TRANSACTIONS OF THE

AMERICAN MATHEMATICAL SOCIETY

Volume 365, Number 2, February 2013, Pages 785-809

S 0002-9947(2012)05648-0

Article electronically published on June 20, 2012

\title{
INTRINSIC VOLUMES OF RANDOM POLYTOPES WITH VERTICES ON THE BOUNDARY OF A CONVEX BODY
}

\author{
KÁROLY J. BÖRÖCZKY, FERENC FODOR, AND DANIEL HUG
}

\begin{abstract}
Let $K$ be a convex body in $\mathbb{R}^{d}$, let $j \in\{1, \ldots, d-1\}$, and let $\varrho$ be a positive and continuous probability density function with respect to the $(d-1)$-dimensional Hausdorff measure on the boundary $\partial K$ of $K$. Denote by $K_{n}$ the convex hull of $n$ points chosen randomly and independently from $\partial K$ according to the probability distribution determined by $\varrho$. For the case when $\partial K$ is a $C^{2}$ submanifold of $\mathbb{R}^{d}$ with everywhere positive Gauss curvature, M. Reitzner proved an asymptotic formula for the expectation of the difference of the $j$ th intrinsic volumes of $K$ and $K_{n}$, as $n \rightarrow \infty$. In this article, we extend this result to the case when the only condition on $K$ is that a ball rolls freely in $K$.
\end{abstract}

\section{INTRODUCTION}

Random polytopes in Euclidean space $\mathbb{R}^{d}$ can be defined in various ways. If $x_{1}, \ldots, x_{n}$ are $n$ random points sampled from a given convex body $K \subset \mathbb{R}^{d}$, then the convex hull of these random points yields a random polytope which has been studied extensively in the literature. The present focus is on a related though different model of a random polytope which has not been explored to the same extent. Instead of choosing the points from all of $K$, we sample random points from the boundary of $K$. The convex hull of these points then provides a model of a random polytope which will be considered here. Our main focus is on the convergence of the expectation of geometric functionals (intrinsic volumes) of such a random polytope. The main result, stated in Theorem 1.2, extends previous work by relaxing the regularity assumptions on $K$. This is a nontrivial task, since the speed of convergence depends in a crucial way on the boundary structure of $K$, in particular, on the (generalized) curvatures, of $K$. The present approach refines arguments that have recently been developed in [4] to establish first order results for the aforementioned model of a random polytope, and it combines geometric and probabilistic ideas.

Before stating our results explicitly, we provide the required background and notation. Our basic setting is the $d$-dimensional Euclidean space $\mathbb{R}^{d}, d \geq 2$, with scalar product $\langle\cdot, \cdot\rangle$ and norm $\|\cdot\|$. By $\mathcal{H}^{j}$ we denote the $j$-dimensional Hausdorff measure, where $\mathcal{H}^{d}$ is simply called the volume $V_{d}$. Let $B^{j}$ be the unit ball of $\mathbb{R}^{j}$

Received by the editors March 23, 2011.

2010 Mathematics Subject Classification. Primary 52A22; Secondary 60D05, 52A27.

The first author was supported by OTKA grant 75016, and by the EU Marie Curie FP7 IEF grant GEOSUMSETS.

The second author was supported by Hungarian OTKA grants 68398 and 75016 and by the János Bolyai Research Scholarship of the Hungarian Academy of Sciences. 
with center at the origin, and let $S^{j-1}$ be its boundary. Then we write $\alpha_{j}=\mathcal{H}^{j}\left(B^{j}\right)$ for the $j$-dimensional volume of $B^{j}$, and hence $\mathcal{H}^{j-1}\left(S^{j-1}\right)=j \alpha_{j}$ is the surface content of $B^{j}$. The relative boundary of a compact convex set $C \subset \mathbb{R}^{d}$ is denoted by $\partial C$. Finally, the convex hull of subsets $X_{1}, \ldots, X_{r}$ and points $z_{1}, \ldots, z_{s}$ is denoted by $\left[X_{1}, \ldots, X_{r}, z_{1}, \ldots, z_{s}\right]$.

Throughout this paper, $K$ is a convex body (compact convex set) with interior points in $\mathbb{R}^{d}$; for notions of convexity we follow the monographs by Schneider [18] or Gruber 8 . The boundary of $K$ is denoted by $\partial K$. We say that $\partial K$ is twice differentiable in the generalized sense at a boundary point $x \in \partial K$ if there exists a positive semi-definite quadratic form $Q$ on $\mathbb{R}^{d-1}$, the so-called second fundamental form, with the following property: If $K$ is positioned in such a way that $x=o$ and $\mathbb{R}^{d-1}$ is a support hyperplane of $K$, then in a neighborhood of the origin $o, \partial K$ is the graph of a convex function $f$ defined on a $(d-1)$-dimensional ball around $o$ in $\mathbb{R}^{d-1}$ satisfying

$$
f(z)=\frac{1}{2} Q(z)+o\left(\|z\|^{2}\right)
$$

as $z \rightarrow o$. According to a classical result of Alexandrov (see P.M. Gruber 8 or R. Schneider [18]), $\partial K$ is twice differentiable in the generalized sense at $\mathcal{H}^{d-1}$ almost all points $x \in \partial K$. Such boundary points are also called normal boundary points. We write $k_{1}(x), \ldots, k_{d-1}(x)$ for the (generalized) principal curvatures of $\partial K$ at $x \in \partial K$, which are just the eigenvalues of $Q$. Furthermore, $H_{j}(x)$ denotes the normalized $j$ th elementary symmetric function of the principal curvatures of $\partial K$ at the normal boundary point $x$. Here the dependence of this function on $K$ is not made explicit. Thus, for $j \in\{1, \ldots, d-1\}$, we have

$$
H_{j}(x)=\left(\begin{array}{c}
d-1 \\
j
\end{array}\right)^{-1} \sum_{1 \leq i_{1}<\cdots<i_{j} \leq d-1} k_{i_{1}}(x) \cdots k_{i_{j}}(x),
$$

and this definition is supplemented by $H_{0}(x):=1$. In particular, $H_{d-1}(x)$ is the Gaussian curvature and $H_{1}(x)$ is the mean curvature of $\partial K$ at $x$. We say that $\partial K$ is $C_{+}^{k}$, for some $k \geq 2$, if $\partial K$ is a $C^{k}$ submanifold of $\mathbb{R}^{d}$ and its Gaussian curvature is positive everywhere.

The intrinsic volumes $V_{j}(K), j=0, \ldots, d$, of a convex body $K \subset \mathbb{R}^{d}$ can be introduced as coefficients of the Steiner formula

$$
V_{d}\left(K+\lambda B^{d}\right)=\sum_{j=0}^{d} \lambda^{d-j} \alpha_{d-j} V_{j}(K),
$$

where $K+\lambda B^{d}$ is the Minkowski sum of $K$ and the ball $\lambda B^{d}$ of radius $\lambda \geq 0$. In particular, $V_{d}$ is the volume functional, $V_{0}(K)=1, V_{1}$ is proportional to the mean width and $V_{d-1}$ is a multiple of the surface area. Alternately, intrinsic volumes can be obtained as mean projection volumes. Specifically, for $j=1, \ldots d-1$, it is well known that

$$
V_{j}(K)=\frac{\left(\begin{array}{l}
d \\
j
\end{array}\right) \alpha_{d}}{\alpha_{j} \alpha_{d-j}} \int_{\mathcal{L}_{j}^{d}} V_{j}(K \mid L) \nu_{j}(d L),
$$

where $\mathcal{L}_{j}^{d}$ is the Grassmannian of all $j$-dimensional linear subspaces of $\mathbb{R}^{d}$ equipped with the (unique) Haar probability measure $\nu_{j}$ and, for $L \in \mathcal{L}_{j}^{d}, K \mid L$ denotes the orthogonal projection of $K$ onto $L$. Here, $V_{j}(K \mid L)$ is just the $j$-dimensional volume (Lebesgue measure) of $K \mid L$. 
We say that a ball rolls freely in a convex body $K \subset \mathbb{R}^{d}$ if there exists some $r>0$ such that any $x \in \partial K$ lies on the boundary of some Euclidean ball $B$ of radius $r$ with $B \subset K$. The existence of a rolling ball is equivalent to saying that the exterior unit normal is a Lipschitz map on $\partial K$ (see D. Hug [14]). In particular, W. Blaschke observed that if $\partial K$ is $C^{2}$, then $K$ has a rolling ball (see D. Hug [14] or K. Leichtweiss [15]). In turn, we say that $K$ rolls freely in a ball of radius $R>0$ if any $x \in \partial K$ lies on the boundary of some Euclidean ball $B$ of radius $R$ with $K \subset B$.

In this paper, we shall consider the following probability model. Let $K$ be a convex body with a rolling ball of radius $r$. Let $\varrho$ be a continuous, positive probability density function defined on $\partial K$; throughout this paper this density is always considered with respect to the boundary measure on $\partial K$. Select the points $x_{1}, \ldots, x_{n}$ randomly and independently from $\partial K$ according to the probability distribution determined by $\varrho$. The convex hull $K_{n}:=\left[x_{1}, \ldots, x_{n}\right]$ then is a random polytope inscribed in $K$. We are going to study the expectation of intrinsic volumes of $K_{n}$. In order to indicate the dependence on the probability density $\varrho$, we write $\mathbb{P}_{\varrho}$ to denote the probability of an event in this probability space and $\mathbb{E}_{\varrho}$ to denote the expected value. For a convex body $K$, the expected value $\mathbb{E}_{\varrho}\left(V_{j}\left(K_{n}\right)\right)$ of the $j$ th intrinsic volume of $K_{n}$ tends to $V_{j}(K)$ as $n$ tends to infinity. It is clear that the asymptotic behavior of $V_{j}(K)-\mathbb{E}_{\varrho}\left(V_{j}\left(K_{n}\right)\right)$ is determined by the shape of the boundary of $K$. The asymptotic behavior in the case when the boundary of $K$ is a $C_{+}^{2}$ submanifold of $\mathbb{R}^{d}$, was described by M. Reitzner in [16].

Theorem 1.1 (Reitzner, 2002). Let $K$ be a convex body in $\mathbb{R}^{d}$ with $C_{+}^{2}$ boundary, and let $\varrho$ be a continuous, positive probability density function on $\partial K$. Denote by $\mathbb{E}_{\varrho}\left(V_{j}\left(K_{n}\right)\right), j=1, \ldots, d$, the expected $j$ th intrinsic volume of the convex hull of $n$ random points on $\partial K$ chosen independently and according to the density function @. Then

$$
V_{j}(K)-\mathbb{E}_{\varrho}\left(V_{j}\left(K_{n}\right)\right) \sim c^{(j, d)} \int_{\partial K} \varrho(x)^{-\frac{2}{d-1}} H_{d-1}(x)^{\frac{1}{d-1}} H_{d-j}(x) \mathcal{H}^{d-1}(d x) \cdot n^{-\frac{2}{d-1}}
$$

as $n \rightarrow \infty$, where the constant $c^{(j, d)}$ only depends on $j$ and dimension $d$.

For $j=d$, in the case of the volume functional, C. Schütt and E. Werner [21] extended (1.2) to any convex body $K$ such that a ball of radius $r$ rolls freely in $K$ and, in addition, $K$ rolls freely in a ball of radius $R$, for some $R>r>0$. The latter assumption of $K$ rolling freely inside a ball implies a uniform lower bound for the principle curvatures of $\partial K$ whenever they exist. They also calculated the constant $c^{(d, d)}$ explicitly, that is,

$$
c^{(d, d)}=\frac{(d-1)^{\frac{d+1}{d-1}} \Gamma\left(d+1+\frac{2}{d-1}\right)}{2(d+1) !\left[(d-1) \alpha_{d-1}\right]^{\frac{2}{d-1}}} .
$$

Moreover, C. Schütt and E. Werner [21] showed that for fixed $K$, the minimum of the integral expression in (1.2) is attained for the probability density function

$$
\varrho_{0}(x)=\frac{H_{d-1}(x)^{\frac{1}{d+1}}}{\int_{\partial K} H_{d-1}(x)^{\frac{1}{d+1}} \mathcal{H}^{d-1}(d x)} .
$$


Our main goal is to extend Theorem[1.1 to the case where $K$ is only assumed to have a rolling ball, for all $j=1, \ldots, d$. In particular, the Gauss curvature is allowed to be zero on a set of positive boundary measure. More explicitly, we shall prove

Theorem 1.2. The asymptotic formula (1.2) holds if $K$ is a convex body in $\mathbb{R}^{d}$ in which a ball rolls freely.

The present method of proof for Theorem 1.2 is different from the one used by Reitzner [16] or Schütt and Werner [21. It is inspired by the arguments from our previous paper [4] concerning random points chosen from a convex body, however, the case of random points chosen from the boundary is more delicate.

Examples show that in general the condition that a ball rolls freely inside $K$ cannot be dropped in Theorem 1.2, For the mean width, general bounds are provided in the following theorem.

Theorem 1.3. Let $K$ be a convex body in $\mathbb{R}^{d}$, and let $\varrho$ be a continuous, positive probability density function on $\partial K$. Then there exist positive constants $c_{1}, c_{2}$, depending on $K$ and $\varrho$, such that for any $n \geq d+1$,

$$
c_{1} n^{-\frac{2}{d-1}} \leq \mathbb{E}_{\varrho}\left(V_{1}(K)-V_{1}\left(K_{n}\right)\right) \leq c_{2} n^{-\frac{1}{d-1}} .
$$

The lower bound is of optimal order if $K$ has a rolling ball, and the upper bound is of optimal order, if $K$ is a polytope.

For comparison, let us review the main known results about the convex hull $K(n)$ of $n$ points chosen randomly, independently and uniformly from $K$. In the case where a ball rolls freely inside $K$, the analogue of Theorem 1.2 is established in K. Böröczky Jr., L. M. Hoffmann and D. Hug [3]. For the case of the volume functional and an arbitrary convex body K, C. Schütt [19] proved (see K.J. Böröczky, F. Fodor, D. Hug [4] for some corrections and an extension) that

$$
\lim _{n \rightarrow \infty} n^{\frac{2}{d+1}}\left(V_{d}(K)-\mathbb{E}\left(V_{d}(K(n))\right)=c_{d} V_{d}(K)^{\frac{2}{d+1}} \int_{\partial K} H_{d-1}(x)^{\frac{1}{d+1}} \mathcal{H}^{d-1}(d x),\right.
$$

where the constant $c_{d}>0$ only depends on the dimension $d$ and is explicitly known. Concerning the order of approximation, we have

$$
\begin{gathered}
\gamma_{1} n^{-2 /(d+1)}<V_{1}(K)-\mathbb{E} V_{1}(K(n))<\gamma_{2} n^{-1 / d}, \\
\gamma_{3} n^{-1} \ln ^{d-1} n<V_{d}(K)-\mathbb{E} V_{d}(K(n))<\gamma_{4} n^{-2 /(d+1)},
\end{gathered}
$$

where $\gamma_{1}, \ldots, \gamma_{4}>0$ are constants which may depend on $K$. The inequalities (1.3) are due to R. Schneider [17, and (1.4) is due to I. Bárány and D. Larman [2]. The orders are best possible, being attained in (1.3) (left) and (1.4) (right) by sufficiently smooth bodies, and in (1.3) (right) and (1.4) (left) by polytopes.

The proof of Theorem 1.2 is given in the following three sections. In Section 2, we rewrite the difference $V_{j}(K)-\mathbb{E}_{\varrho}\left(V_{j}\left(K_{n}\right)\right)$ in an integral geometric way. The inner integral involved in this integral geometric description is extended over the projection $K \mid L$ of $K$ to $L$, where $L$ is a $j$-dimensional linear subspace. Then we show that, up to an error term of lower order, the main contribution comes from a neighborhood of the boundary $\partial(K \mid L)$, where this neighborhood is shrinking at a well-defined speed $t(n):=n^{-1 /(d-1)}$ as $n \rightarrow \infty$. Further application of an integral geometric decomposition then shows that the proof boils down to determining the limit

$$
\lim _{n \rightarrow \infty} \int_{0}^{t(n)} n^{\frac{2}{d-1}}\langle y, u(y)\rangle \mathbb{P}_{\varrho}\left(y_{t} \notin K_{n} \mid L\right) d t,
$$


where $y \in \partial(K \mid L)$ is a point for which there exists a normal boundary point $x$ of $K$ such that the orthogonal projection of $x$ onto $L$ is $y$, that is, $y=x \mid L$. Moreover, $u(y) \in L$ denotes the unique exterior unit normal to $\partial(K \mid L)$ at $y \in \partial(K \mid L)$ with respect to $L$ as ambient space and $y_{t}:=(1-t) y$.

In order to determine the limit, we distinguish two cases. The case where the Gauss curvature of $K$ at $x$ is zero is treated directly. In Section 3, we deal with the case of positive Gauss curvature. In a first step, we choose a reparametrization of the integral which relates the parameter $t$ to the probability content $s$ of that part of the boundary of $K$ near $x$ that is cut off by a cap determined by the parameter $t$. This reparametrization has the effect of extracting the relevant geometric information from $K$. What remains to be shown is that the transformed integrals are essentially independent of $K$ and yield the same value for the unit ball with the uniform probability density on its boundary. This latter step is divided into two lemmas in Section 3. Whereas both lemmas have analogues in our previous work 4, the present arguments are more delicate and the second lemma has to be established by reasoning different from that in 4. The proof is then completed in Section 4, where, in addition to the previous steps, a very special case of Theorem 1.1 is employed ( $K$ being the unit ball) as well as an integral geometric lemma from [3]. The final section is devoted to a proof of Theorem 1.3.

\section{General estimates}

In order to prove Theorem 1.2 , we start by rewriting $V_{j}(K)-\mathbb{E}_{\varrho}\left(V_{j}\left(K_{n}\right)\right)$ in an integral geometric form. For this, we use Kubota's formula and Fubini's theorem to obtain

$$
\begin{aligned}
& V_{j}(K)-\mathbb{E}_{\varrho}\left(V_{j}\left(K_{n}\right)\right) \\
& =\int_{\partial K} \ldots \int_{\partial K}\left(V_{j}(K)-V_{j}\left(K_{n}\right)\right) \prod_{i=1}^{n} \varrho\left(x_{i}\right) \mathcal{H}^{d-1}\left(d x_{1}\right) \ldots \mathcal{H}^{d-1}\left(d x_{n}\right) \\
& =\frac{\left(\begin{array}{l}
d \\
j
\end{array}\right) \alpha_{d}}{\alpha_{j} \alpha_{d-j}} \int_{\partial K} \ldots \int_{\partial K} \int_{\mathcal{L}_{j}^{d}}\left(V_{j}(K \mid L)-V_{j}\left(K_{n} \mid L\right)\right) \\
& \quad \times \prod_{i=1}^{n} \varrho\left(x_{i}\right) \nu_{j}(d L) \mathcal{H}^{d-1}\left(d x_{1}\right) \ldots \mathcal{H}^{d-1}\left(d x_{n}\right) \\
& =\frac{\left(\begin{array}{l}
d \\
j
\end{array}\right) \alpha_{d}}{\alpha_{j} \alpha_{d-j}} \int_{\mathcal{L}_{j}^{d}} \int_{L} \int_{\partial K} \ldots \int_{\partial K} \mathbf{1}\left\{y \in K \mid L \text { and } y \notin K_{n} \mid L\right\} \\
& \quad \times \prod_{i=1}^{n} \varrho\left(x_{i}\right) \mathcal{H}^{d-1}\left(d x_{1}\right) \ldots \mathcal{H}^{d-1}\left(d x_{n}\right) \mathcal{H}^{j}(d y) \nu_{j}(d L) \\
& =\frac{\left(\begin{array}{l}
d \\
j
\end{array}\right) \alpha_{d}}{\alpha_{j} \alpha_{d-j}} \int_{\mathcal{L}_{j}^{d}} \int_{K \mid L} \mathbb{P}_{\varrho}\left(y \notin K_{n} \mid L\right) \mathcal{H}^{j}(d y) \nu_{j}(d L) .
\end{aligned}
$$

Now we introduce some geometric tools. If $K$ has a rolling ball of radius $r$, then so does $K \mid L$ for any $L \in \mathcal{L}_{j}^{d}$. Furthermore, $\partial K$ has a unique outer unit normal vector $u(x)$ at each boundary point $x \in \partial K$. If $L \in \mathcal{L}_{j}^{d}, y \in \partial(K \mid L)$ and $x \in K$ are such that $y=x \mid L$, then $x \in \partial K$ and the outer unit normal of $\partial(K \mid L)$ at $y$ is equal to $u(x)$. Here $x \mid L$ denotes the orthogonal projection of $x$ onto $L$. 
Since the statement of the theorem is translation invariant, we may assume that

$$
r B^{d} \subset K \subset R B^{d}
$$

for some $R>0$. For $t \in(0,1)$, let $K_{t}:=(1-t) K$, and for $x \in \partial K$, let $x_{t}:=(1-t) x$. Similarly, $(K \mid L)_{t}:=(1-t)(K \mid L)$ and $y_{t}:=(1-t) y$ for $y \in \partial(K \mid L)$.

For $x \in \partial K$ and $t \in(0,1)$, let

$$
x_{t}^{*}:=x-\langle t x, u(x)\rangle u(x) .
$$

If $t \in\left(0, \frac{r}{R}\right)$, then (2.2) implies that

$$
\operatorname{tr} \leq\left\langle x-x_{t}^{*}, u(x)\right\rangle=\left\langle x-x_{t}, u(x)\right\rangle<r .
$$

The existence of a rolling ball at $x$ yields that if $t \in\left(0, \frac{r}{R}\right)$, then

$$
x_{t}^{*}+r \sqrt{t}\left(u(x)^{\perp} \cap B^{d}\right) \subset K .
$$

On the other hand, we have

$$
\left\|x_{t}^{*}-x_{t}\right\|<R t .
$$

For real functions $f$ and $g$ defined on the same space, we write $f \ll g$ or $f=O(g)$ if there exists a positive constant $\gamma$, depending only on $K$ and $\varrho$, such that $|f| \leq \gamma \cdot g$.

We shall use the notion of a "coordinate corner". Given an orthonormal basis in a linear $i$-dimensional subspace $L$, the corresponding $(i-1)$-dimensional coordinate planes cut $L$ into $2^{i}$ convex cones, which we call coordinate corners (with respect to $L$ and the given basis). In the following, we write $\gamma_{1}, \gamma_{2}, \ldots$ for positive constants which merely depend on $K$ and $\varrho$.

Let us estimate the probability that $o \notin K_{n}$. There exists a constant $\gamma_{1}>0$ such that the probability content of each of the parts of $\partial K$ contained in one of the $2^{d}$ coordinate corners of $\mathbb{R}^{d}$ is at least $\gamma_{1}$. Now if $o \notin K_{n}$, then $o$ can be strictly separated from $K_{n}$ by a hyperplane. It follows that $\left\{x_{1}, \ldots, x_{n}\right\}$ is disjoint from one of these coordinate corners, and hence

$$
\mathbb{P}\left(o \notin K_{n}\right) \leq 2^{d}\left(1-\gamma_{1}\right)^{n} .
$$

This fact will be used, for instance, in the proof of the subsequent lemma. In the following, for $x \in \mathbb{R}^{d}$ we use the shorthand notation $\mathbb{R}_{+} x:=\{\lambda x: \lambda \geq 0\}$.

Lemma 2.1. There exist constants $\delta, \gamma_{2} \in(0,1)$, depending on $K$ and $\varrho$, such that if $L \in \mathcal{L}_{j}^{d}, y \in \partial(K \mid L)$ and $t \in(0, \delta)$, then

$$
\mathbb{P}_{\varrho}\left(y_{t} \notin K_{n} \mid L\right) \ll\left(1-\gamma_{2} t^{\frac{d-1}{2}}\right)^{n} .
$$

Proof. Let $y \in \partial(K \mid L)$ and $x \in \partial K$ be such that $y=x \mid L$. Let $\Theta_{1}^{\prime}, \ldots, \Theta_{2^{d-1}}^{\prime}$ be the coordinate corners with respect to some basis vectors in $u(x)^{\perp}$. In addition, for $i=1, \ldots, 2^{d-1}$ and $t \in(0,1)$, let

$$
\Theta_{i, t}=\partial K \cap\left(x_{t}+\left[\Theta_{i}^{\prime}, \mathbb{R}_{+} x\right]\right) .
$$

Since $\varrho$ is positive and continuous, we have

$$
\int_{\Theta_{i, t}} \varrho(x) \mathcal{H}^{d-1}(d x) \geq \gamma_{3} \mathcal{H}^{d-1}\left(\Theta_{i, t}\right) .
$$

If $y_{t} \notin K_{n} \mid L$ and $o \in K_{n}$, then there exists a $(j-1)$-dimensional affine plane $H_{L}$ in $L$ through $y_{t}$, bounding the half-spaces $H_{L}^{-}$and $H_{L}^{+}$in $L$, for which $K_{n} \mid L \subset H_{L}^{-}$. Now, if $L^{\perp}$ is the orthogonal complement of $L$ in $\mathbb{R}^{d}$, then $H:=H_{L}+L^{\perp}$ is a hyperplane 
in $\mathbb{R}^{d}$ with the property that $x_{t} \in H$ and $K_{n} \subset H^{-}:=H_{L}^{-}+L^{\perp}$. Furthermore, $\Theta_{i, t} \subset H^{+}:=H_{L}^{+}+L^{\perp}$ for some $i \in\left\{1, \ldots, 2^{d-1}\right\}$, because $o \in K_{n} \subset H^{-}$. Therefore

$$
\mathbb{P}_{\varrho}\left(y_{t} \notin K_{n} \mid L, o \in K_{n}\right) \leq \sum_{i=1}^{2^{d-1}}\left(1-\gamma_{3} \mathcal{H}^{d-1}\left(\Theta_{i, t}\right)\right)^{n} .
$$

Combining (2.4) and (2.5), we deduce the existence of a constant $\gamma_{4}>0$ such that if $t \leq \gamma_{4}$, then the orthogonal projection of $\Theta_{i, t}$ into $u(x)^{\perp}$ contains a translate of $\Theta_{i}^{\prime} \cap(r / 2) \sqrt{t} B^{d}$, and therefore

$$
\mathcal{H}^{d-1}\left(\Theta_{i, t}\right) \geq \gamma_{5} t^{\frac{d-1}{2}}
$$

for $i=1, \ldots, 2^{d-1}$. In turn, we obtain

$$
\mathbb{P}_{\varrho}\left(y_{t} \notin K_{n} \mid L, o \in K_{n}\right) \ll\left(1-\gamma_{6} t^{\frac{d-1}{2}}\right)^{n} .
$$

On the other hand, if $o \notin K_{n} \mid L$, then (2.6) holds. Combining this with (2.7), we conclude the proof of the lemma.

Subsequently, the estimate of Lemma 2.1 will be used, for instance, to restrict the domain of integration (cf. Lemma 2.3) and to justify an application of Lebesgue's dominated convergence theorem (see (2.12) ). For these applications, we also need that, if $x \in \partial K$ and $c>0$ satisfies $\bar{\omega}:=c \delta^{\frac{d-1}{2}}<1$, then

$$
\int_{0}^{\delta}\left(1-c t^{\frac{d-1}{2}}\right)^{n} d t=c^{\frac{-2}{d-1}} \frac{2}{d-1} \int_{0}^{\bar{\omega}} s^{\frac{2}{d-1}-1}(1-s)^{n} d s \ll c^{\frac{-2}{d-1}} \cdot n^{\frac{-2}{d-1}},
$$

where we use that $(1-s)^{n} \leq e^{-n s}$ for $s \in[0,1]$ and $n \in \mathbb{N}$.

The next lemma will allow us to decompose integrals in a suitable way. We write $u(y) \in L$ to denote the unique exterior unit normal to $\partial(K \mid L)$ at $y \in \partial(K \mid L)$ with respect to $L$ as ambient space. It will always be clear from the context whether we mean the exterior unit normal at a point $x \in \partial K$ or at a point $y \in \partial(K \mid L)$. In the next lemma, $\delta$ is chosen as in Lemma 2.1.

Lemma 2.2. If $0 \leq t_{0}<t_{1}<\delta$ and $h: K \mid L \rightarrow[0, \infty]$ is a measurable function, then

$$
\begin{aligned}
\int_{(K \mid L)_{t_{0}} \backslash(K \mid L)_{t_{1}}} \mathbb{P}_{\varrho}\left(x \notin K_{n} \mid L\right) h(x) \mathcal{H}^{j}(d x) \\
=\int_{\partial(K \mid L)} \int_{t_{0}}^{t_{1}}(1-t)^{j-1} \mathbb{P}_{\varrho}\left(y_{t} \notin K_{n} \mid L\right)\langle y, u(y)\rangle h\left(y_{t}\right) d t \mathcal{H}^{j-1}(d y) .
\end{aligned}
$$

Proof. The set $\partial(K \mid L)$ is a $(j-1)$-dimensional submanifold of $L$ of class $C^{1}$, and the map

$$
T: \partial(K \mid L) \times\left(t_{0}, t_{1}\right) \rightarrow \operatorname{int}(K \mid L)_{t_{0}} \backslash(K \mid L)_{t_{1}}, \quad(y, t) \mapsto y_{t},
$$

is a $C^{1}$ diffeomorphism with Jacobian $J T(y, t)=(1-t)^{j-1}\langle y, u(y)\rangle \geq 0$. Thus the assertion follows from Federer's area/coarea theorem (see [7]).

In the following, we use the abbreviation $t(n):=n^{\frac{-1}{d-1}}$.

Lemma 2.3. Let $1 \leq j \leq d-1$. Then we have

$$
\int_{\mathcal{L}_{j}^{d}} \int_{(K \mid L)_{t(n)}} \mathbb{P}_{\varrho}\left(y \notin K_{n} \mid L\right) \mathcal{H}^{j}(d y) \nu_{j}(d L)=o\left(n^{\frac{-2}{d-1}}\right) .
$$


Proof. Let $\delta, \gamma_{2} \in(0,1)$ be chosen as in Lemma 2.1. We may assume that $n$ is large enough to satisfy $t(n)<\delta$ and $n \geq\left(\gamma_{2}\right)^{2}$. First, we treat that part of the integral which extends over the subset $(K \mid L)_{\delta}$ of $(K \mid L)_{t(n)}$.

Let $\omega:=\delta r$. Then (2.3) yields

$$
\left\langle x-x_{\delta}, u(x)\right\rangle \geq \omega \text { for } x \in \partial K .
$$

There exists a constant $\gamma_{7}>0$ such that the probability measure of $\left(x+\frac{\omega}{2} B^{d}\right) \cap \partial K$ is at least $\gamma_{7}$ for all $x \in \partial K$. We choose a maximal set $\left\{z_{1}, \ldots, z_{m}\right\} \subset \partial K$ such that $\left\|z_{i}-z_{l}\right\| \geq \frac{\omega}{2}$ for $i \neq l$.

For $L \in \mathcal{L}_{j}^{d}$, let $y \in(K \mid L)_{\delta}$. If $y \notin K_{n} \mid L$, then there exist a hyperplane $H$ in $\mathbb{R}^{d}$ and a half-space $H^{-}$bounded by $H$ such that $y \in H, H$ is orthogonal to $L$, and $K_{n} \subset \operatorname{int}\left(H^{-}\right)$. Choose $x \in \partial K$ such that $u(x)$ is an exterior unit normal to $H^{-}$. Since $H$ intersects $K_{\delta}$, we have $\langle x-y, u(x)\rangle \geq \omega$ by (2.9). Now there exists some $i \in\{1, \ldots, n\}$ with $\left\|x-z_{i}\right\| \leq \frac{\omega}{2}$, and hence $\left\{x_{1}, \ldots, x_{n}\right\} \subset \operatorname{int}\left(H^{-}\right)$yields that $\left\{x_{1}, \ldots, x_{n}\right\}$ is disjoint from $z_{i}+\frac{\omega}{2} B^{d}$. In particular, we have

$$
\mathbb{P}_{\varrho}\left(y \notin K_{n} \mid L\right) \leq m\left(1-\gamma_{7}\right)^{n} .
$$

Next, let $y \in \partial(K \mid L)$. If $t \in(t(n), \delta)$, then Lemma 2.1 yields

$$
\mathbb{P}_{\varrho}\left(y_{t} \notin K_{n} \mid L\right) \ll\left(1-\gamma_{2} n^{-\frac{1}{2}}\right)^{n}<e^{-\gamma_{2} n^{\frac{1}{2}}} \ll n^{\frac{-3}{d+1}} .
$$

In particular, writing $I$ to denote the integral in Lemma 2.3, we obtain from Lemma 2.2, (2.10) and (2.11) that

$$
\begin{aligned}
I \ll & \int_{\mathcal{L}_{j}^{d}} \int_{(K \mid L)_{\delta}} \mathbb{P}_{\varrho}\left(y \notin K_{n} \mid L\right) \mathcal{H}^{j}(d y) \nu_{j}(d L) \\
& +\int_{\mathcal{L}_{j}^{d}} \int_{t(n)}^{\delta} \int_{\partial(K \mid L)} \mathbb{P}_{\varrho}\left(y_{t} \notin K_{n} \mid L\right) \mathcal{H}^{j-1}(d y) d t \nu_{j}(d L) \\
& \ll \quad m\left(1-\gamma_{7}\right)^{n}+\int_{\mathcal{L}_{j}^{d}} \int_{\partial(K \mid L)} n^{\frac{-3}{d-1}} \mathcal{H}^{j-1}(d y) \nu_{j}(d L)=o\left(n^{\frac{-2}{d-1}}\right),
\end{aligned}
$$

which is the required estimate.

It follows by applying (2.1), Lemma 2.3 and Lemma 2.2 , in this order, that

$$
\begin{aligned}
& \lim _{n \rightarrow \infty} n^{\frac{2}{d-1}}\left(V_{j}(K)-\mathbb{E}_{\varrho}\left(V_{j}\left(K_{n}\right)\right)\right) \\
& =\frac{\left(\begin{array}{c}
d \\
j
\end{array}\right) \alpha_{d}}{\alpha_{j} \alpha_{d-j}} \lim _{n \rightarrow \infty} n^{\frac{2}{d-1}} \int_{\mathcal{L}_{j}^{d}} \int_{K \mid L} \mathbb{P}_{\varrho}\left(y \notin K_{n} \mid L\right) \mathcal{H}^{j}(d y) \nu_{j}(d L) \\
& =\frac{\left(\begin{array}{c}
d \\
j
\end{array}\right) \alpha_{d}}{\alpha_{j} \alpha_{d-j}} \lim _{n \rightarrow \infty} n^{\frac{2}{d-1}} \int_{\mathcal{L}_{j}^{d}} \int_{(K \mid L) \backslash(K \mid L)_{t(n)}} \mathbb{P}_{\varrho}\left(y \notin K_{n} \mid L\right) \mathcal{H}^{j}(d y) \nu_{j}(d L) \\
& =\frac{\left(\begin{array}{c}
d \\
j
\end{array}\right) \alpha_{d}}{\alpha_{j} \alpha_{d-j}} \lim _{n \rightarrow \infty} \int_{\mathcal{L}_{j}^{d}} \int_{\partial(K \mid L)} \int_{0}^{t(n)} n^{\frac{2}{d-1}} \mathbb{P}_{\varrho}\left(y_{t} \notin K_{n} \mid L\right)(1-t)^{j-1}\langle y, u(y)\rangle d t \\
& \mathcal{H}^{j-1}(d y) \nu_{j}(d L) .
\end{aligned}
$$

We deduce from Lemma 2.1 and (2.8) that if $n>n_{0}, L \in \mathcal{L}_{j}^{d}$ and $y \in \partial(K \mid L)$, then

$$
\int_{0}^{t(n)} n^{\frac{2}{d-1}} \mathbb{P}_{\varrho}\left(y_{t} \notin K_{n} \mid L\right)\langle y, u(y)\rangle(1-t)^{j-1} d t \ll C,
$$


where $n_{0}$ and $C$ depend on $K$ and $\varrho$. Therefore, we may apply Lebesgue's dominated convergence theorem, and thus we conclude

$$
\lim _{n \rightarrow \infty} n^{\frac{2}{d-1}}\left(V_{j}(K)-\mathbb{E}_{\varrho}\left(V_{j}\left(K_{n}\right)\right)\right)=\frac{\left(\begin{array}{c}
d \\
j
\end{array}\right) \alpha_{d}}{\alpha_{j} \alpha_{d-j}} \int_{\mathcal{L}_{j}^{d}} \int_{\partial(K \mid L)} J_{\varrho}(y, L) \mathcal{H}^{j-1}(d y) \nu_{j}(d L),
$$

where, for $L \in \mathcal{L}_{j}^{d}$ and $y \in \partial(K \mid L)$, we have

$$
J_{\varrho}(y, L):=\lim _{n \rightarrow \infty} \int_{0}^{t(n)} n^{\frac{2}{d-1}}\langle y, u(y)\rangle \mathbb{P}_{\varrho}\left(y_{t} \notin K_{n} \mid L\right) d t .
$$

Subsequently, we shall inspect this limit more closely. In a first step, we shall consider those points $y \in \partial(K \mid L)$ for which there is a normal boundary point $x \in \partial K$ with $y=x \mid L$ and $H_{d-1}(x)=0$.

Lemma 2.4. Let $L \in \mathcal{L}_{j}^{d}$, and let $y \in \partial(K \mid L)$. If $x \in \partial K$ is a normal boundary point of $K$ with $y=x \mid L$ and $H_{d-1}(x)=0$, then $J_{\varrho}(y, L)=0$.

Proof. Let $x \in \partial K$ be a normal boundary point with $y=x \mid L$ and $H_{d-1}(x)=0$. First, we show the existence of a decreasing function $\varphi$ on $\left(0, \frac{r}{R}\right)$ with $\lim _{t \rightarrow 0^{+}} \varphi(t)=$ $\infty$ satisfying

$$
\mathbb{P}_{\varrho}\left(y_{t} \notin K_{n} \mid L\right) \leq 2^{d-1}\left(1-\varphi(t) t^{\frac{d-1}{2}}\right)^{n} .
$$

In the following, we always assume that $t>0$ is sufficiently small, that is $n$ is sufficiently large, so that all expressions that arise are well defined. Let $v_{1}, \ldots, v_{d-1}$ be an orthonormal basis in $u(x)^{\perp}$ such that these vectors are principal directions of curvature of $K$ at $x$ and such that the curvature is zero in the direction of $v_{1}$. In addition, let $\Theta_{1}^{\prime}, \ldots, \Theta_{2^{d-1}}^{\prime}$ be the coordinate corners in $u(x)^{\perp}$, and, for $i=1, \ldots, 2^{d-1}$ and $t \in(0,1)$, let $\Theta_{i, t}=\partial K \cap\left(x_{t}+\left[\Theta_{i}^{\prime}, \mathbb{R}_{+} x\right]\right)$ as before. The continuity of $\varrho$ yields that

$$
\int_{\Theta_{i, t}} \varrho(x) \mathcal{H}^{d-1}(d x) \gg \mathcal{H}^{d-1}\left(\Theta_{i, t}\right) .
$$

Since the curvature is zero in the direction of $v_{1}$, there exists a function $\psi$ on $\left(0, \frac{r}{R}\right)$ with $\lim _{t \rightarrow 0^{+}} \psi(t)=\infty$ satisfying

$$
x_{t}^{*}-\psi(t) \sqrt{t} v_{1} \in K \text { and } x_{t}^{*}+\psi(t) \sqrt{t} v_{1} \in K .
$$

Combining (2.4) and (2.5), we deduce the existence of a decreasing function $\tilde{\varphi}$ on $\left(0, \frac{r}{R}\right)$ with $\lim _{t \rightarrow 0^{+}} \tilde{\varphi}(t)=\infty$ satisfying

$$
\int_{\Theta_{i, t}} \varrho(x) \mathcal{H}^{d-1}(d x) \geq \tilde{\varphi}(t) t^{\frac{d-1}{2}},
$$

for $i=1, \ldots, 2^{d-1}$.

First, we assume that $y_{t} \notin K_{n} \mid L$ and $o \in K_{n}$. In particular, then we also have $x_{t} \notin K_{n}$, and hence there exists a hyperplane $H$ through $x_{t}$ such that $K_{n}$ lies on one side of $H$. Since $o \in K_{n}$, it follows that $H$ separates $K_{n}$ from some $\Theta_{i, t}$, and therefore

$$
\mathbb{P}_{\varrho}\left(y_{t} \notin K_{n} \mid L, o \in K_{n}\right) \leq 2^{d-1}\left(1-\tilde{\varphi}(t) t^{\frac{d-1}{2}}\right)^{n}
$$


On the other hand, if $o \notin K_{n} \mid L$, then (2.6) holds. Combining this with (2.15), we conclude (2.14). In turn, we deduce from (2.8) that

$$
J_{\varrho}(y, L) \ll \lim _{n \rightarrow \infty} n^{\frac{2}{d-1}} \int_{0}^{t(n)}\left(1-\varphi(t(n)) t^{\frac{d-1}{2}}\right)^{n} d t \ll \lim _{n \rightarrow \infty} \varphi(t(n))^{\frac{-2}{d-1}}=0 .
$$

In the next section, we study the more difficult case of boundary points with positive Gauss curvature.

\section{NORMAL BOUNDARY POINTS AND CAPS}

Let $L \in \mathcal{L}_{j}^{d}$, and let $y \in \partial(K \mid L)$ be such that $y=x \mid L$ for some (uniquely determined) normal boundary point $x \in \partial K$ with $H_{d-1}(x)>0$. We keep $x$ and $y$ fixed throughout this section. First, we reparametrize $x_{t}$ and $y_{t}$ in terms of the probability measure of the corresponding cap of $\partial K$. Using this reparametrization, we show that $J_{\varrho}(y, L)$ essentially depends only on the random points near $x$ (see Lemma 3.1), and then in a second step we pass from the case of a general convex body $K$ to the case of a Euclidean ball.

For $t \in(0,1)$, we consider the hyperplane $H(x, t):=\left\{z \in \mathbb{R}^{d}:\langle u(x), z\rangle=\right.$ $\left.\left\langle u(x), x_{t}\right\rangle\right\}$, the half-space $H^{+}(x, t):=\left\{z \in \mathbb{R}^{d}:\langle u(x), z\rangle \geq\left\langle u(x), x_{t}\right\rangle\right\}$, and the cap $C(x, t):=K \cap H^{+}(x, t)$ whose bounding hyperplane is $H(x, t)$. Next we reparametrize $x_{t}$ in terms of the induced probability measure of the cap $C(x, t)$; namely,

$$
\tilde{x}_{s}:=x_{t} \quad \text { and } \quad \tilde{y}_{s}:=y_{t},
$$

where, for a given sufficiently small $s \geq 0$, the parameter $t \geq 0$ is uniquely determined by the equation

$$
s=\int_{C(x, t) \cap \partial K} \varrho(w) \mathcal{H}^{d-1}(d w) .
$$

Note that $s$ is a strictly increasing and continuous function of $t$. We further define

$$
\widetilde{C}(x, s)=C(x, t) \quad \text { and } \quad \widetilde{H}(x, s)=H(x, t),
$$

where again, for given $s$, the parameter $t$ is determined by (3.1). Observe that $\partial K \cap H^{+}(x, t)=\partial K \cap C(x, t)$. Subsequently, we explore the relation between $s$ and $t$. Let $f: u(x)^{\perp} \rightarrow[0, \infty]$ be a convex function such that the restriction of the map

$$
F: u(x)^{\perp} \rightarrow \mathbb{R}^{d}, \quad z \mapsto x+z-f(z) u(x),
$$

to a neighborhood of $o$ parametrizes $\partial K$ in a neighborhood of $x$. Moreover, we consider the transformations

$$
\Pi: \mathbb{R}^{d} \rightarrow u(x)^{\perp}, \quad y \mapsto y-x-\langle y-x, u(x)\rangle u(x)
$$

and

$$
T: u(x)^{\perp} \times \mathbb{R} \rightarrow u(x)^{\perp} \times \mathbb{R}, \quad\left(z_{1}, \ldots, z_{d-1}, \alpha\right) \mapsto\left(\sqrt{k_{1}} z_{1}, \ldots, \sqrt{k_{d-1}} z_{d-1}, \alpha\right),
$$


where $u(x)^{\perp}$ is considered to be a subset of $u(x)^{\perp} \times\{0\}$ and $k_{i}=k_{i}(x), i=$ $1, \ldots, d-1$ are the principle curvatures of $\partial K$ at $x$. Then we obtain

$$
\begin{aligned}
& \int_{\partial K \cap H^{+}(x, t)} \varrho(w) \mathcal{H}^{d-1}(d w) \\
& =\int_{\Pi\left(\partial K \cap H^{+}(x, t)\right)} \varrho(F(z)) \sqrt{1+\|\nabla f(z)\|^{2}} \mathcal{H}^{d-1}(d z) \\
& =\int_{T\left(\Pi\left(\partial K \cap H^{+}(x, t)\right)\right)} \varrho\left(F \circ T^{-1}(z)\right) \sqrt{1+\left\|\nabla f\left(T^{-1}(z)\right)\right\|^{2}} H_{d-1}(x)^{-1 / 2} \mathcal{H}^{d-1}(d z) .
\end{aligned}
$$

Let $\bar{K}:=T(K-x)+x$, and hence $T\left(\Pi\left(\partial K \cap H^{+}(x, t)\right)\right)=\Pi\left(\partial \bar{K} \cap H^{+}(x, t)\right)$. If $\bar{f}$ is defined for $\bar{K}$ as $f$ is defined for $K$, and

$$
\bar{\varrho}(w):=\varrho\left(F \circ T^{-1} \circ \Pi(w)\right), \quad g(w):=\frac{\sqrt{1+\left\|\nabla f\left(T^{-1}(\Pi(w))\right)\right\|^{2}}}{\sqrt{1+\|\nabla \bar{f}(\Pi(w))\|^{2}}},
$$

for $w \in \partial \bar{K} \cap H^{+}(x, t)$, then we obtain

$$
\int_{\partial K \cap H^{+}(x, t)} \varrho(w) \mathcal{H}^{d-1}(d w)=H_{d-1}(x)^{-1 / 2} \int_{\partial \bar{K} \cap H^{+}(x, t)} \bar{\varrho}(w) g(w) \mathcal{H}^{d-1}(d w) .
$$

Next we put $H(r):=x-r u(x)+u(x)^{\perp}$ and denote by $n_{\bar{K}}(w)$ the exterior unit normal of $\bar{K}$ at $w \in \partial \bar{K}$. Since (cf. the notes for Section 1.5 (2) in [18])

$\bar{f}(z)=\frac{1}{2}\|z\|^{2}+o\left(\|z\|^{2}\right), \quad\|\nabla \bar{f}(z)\|=\|z\|+o(\|z\|), \quad n_{\bar{K}}(w)=\frac{\nabla \bar{f}(\bar{w})+u(x)}{\sqrt{1+\|\nabla \bar{f}(\bar{w})\|^{2}}}$

with $\bar{w}:=\Pi(w)$ and $z \in u(x)^{\perp}$, we get

$$
{\sqrt{1-\left\langle n_{\bar{K}}(w), u(x)\right\rangle^{2}}}^{-1}=\frac{\sqrt{1+(\|\bar{w}\|+o(\|\bar{w}\|))^{2}}}{\|\bar{w}\|+o(\|\bar{w}\|)} .
$$

Thus a simple application of the coarea formula yields that, for $t>0$ sufficiently small and $d \geq 2$,

$$
\begin{aligned}
& \int_{\partial K \cap H^{+}(x, t)} \varrho(w) \mathcal{H}^{d-1}(d w) \\
& =H_{d-1}(x)^{-1 / 2} \int_{0}^{t\langle x, u(x)\rangle} \int_{\partial \bar{K} \cap H(r)} \bar{\varrho}(w) g(w){\sqrt{1-\left\langle n_{\bar{K}}(w), u(x)\right\rangle^{2}}}^{-1} \mathcal{H}^{d-2}(d w) d r .
\end{aligned}
$$

Since also $\bar{K}$ has a rolling ball, the map $w \mapsto n_{\bar{K}}(w)$ is continuous, and therefore also

$$
r \mapsto \int_{\partial \bar{K} \cap H(r)} \bar{\varrho}(w) g(w){\sqrt{1-\left\langle n_{\bar{K}}(w), u(x)\right\rangle^{2}}}^{-1} \mathcal{H}^{d-2}(d w)
$$

is continuous. This implies that

$$
\begin{aligned}
& \frac{\partial}{\partial t} \int_{\partial K \cap H^{+}(x, t)} \varrho(w) \mathcal{H}^{d-1}(d w) \\
& =\frac{\langle x, u(x)\rangle}{H_{d-1}(x)^{1 / 2}} \int_{\partial \bar{K} \cap H(t\langle x, u(x)\rangle)} \bar{\varrho}(w) g(w){\sqrt{1-\left\langle n_{\bar{K}}(w), u(x)\right\rangle^{2}}}^{-1} \mathcal{H}^{d-2}(d w) \\
& =\frac{\langle x, u(x)\rangle}{H_{d-1}(x)^{1 / 2}} \int_{\partial \bar{K} \cap H(t\langle x, u(x)\rangle)} \bar{\varrho}(w) g(w) \frac{\sqrt{1+(\sqrt{2 t\langle x, u(x)\rangle}+o(\sqrt{t}))^{2}}}{\sqrt{2 t\langle x, u(x)\rangle}+o(\sqrt{t})} \mathcal{H}^{d-2}(d w) .
\end{aligned}
$$


Clearly, we have $\bar{\varrho}(w) \rightarrow \bar{\varrho}(x)=\varrho(x)$ and $g(w) \rightarrow 1$, as $t \rightarrow 0^{+}$, uniformly with respect to $w \in \partial \bar{K} \cap H(t\langle x, u(x)\rangle)$. Moreover, since

$$
\bar{\Gamma}:=\left\{x+z-\frac{1}{2}\|z\|^{2} u(x): z \in u(x)^{\perp}\right\}
$$

is the osculating paraboloid of $\bar{K}$ and $\bar{\Gamma}$ has rotational symmetry, we obtain for $s=s(t)$ that

$$
\begin{aligned}
\lim _{t \rightarrow 0^{+}} t^{-\frac{d-3}{2}} \cdot \frac{\partial s}{\partial t}(t) & =\frac{\varrho(x)\langle x, u(x)\rangle}{H_{d-1}(x)^{1 / 2}} \lim _{t \rightarrow 0^{+}}\left(t^{-\frac{d-3}{2}}(d-1) \alpha_{d-1} \frac{\sqrt{2 t\langle x, u(x)\rangle}^{d-2}}{\sqrt{2 t\langle x, u(x)\rangle}}\right) \\
& =(d-1) \alpha_{d-1} H_{d-1}(x)^{-\frac{1}{2}} \varrho(x)(2\langle x, u(x)\rangle)^{\frac{d-3}{2}}\langle x, u(x)\rangle \\
& =(d-1) \alpha_{d-1} \varrho(x) 2^{\frac{d-3}{2}}\langle x, u(x)\rangle^{\frac{d-1}{2}} H_{d-1}(x)^{-\frac{1}{2}} .
\end{aligned}
$$

Thus we have shown that

$$
\lim _{t \rightarrow 0^{+}} t^{-\frac{d-3}{2}} \cdot \frac{\partial s}{\partial t}(t)=(d-1) \cdot \varrho(x) 2^{\frac{d-3}{2}}\langle x, u(x)\rangle^{\frac{d-1}{2}} H_{d-1}(x)^{-\frac{1}{2}} \alpha_{d-1} .
$$

In the same way, we also obtain

$$
\lim _{t \rightarrow 0^{+}} t^{-\frac{d-1}{2}} \cdot s(t)=\varrho(x) 2^{\frac{d-1}{2}}\langle x, u(x)\rangle^{\frac{d-1}{2}} H_{d-1}(x)^{-\frac{1}{2}} \alpha_{d-1} .
$$

Observe that (3.3) and (3.4) are valid also for $d=2$. In particular, (3.3) and (3.4) imply that $J_{\varrho}(y, L)$ can be rewritten as (cf. (2.13)

$$
J_{\varrho}(y, L)=(d-1)^{-1} G(x)^{2} \lim _{n \rightarrow \infty} \int_{0}^{\zeta(y, n)} n^{\frac{2}{d-1}} \mathbb{P}_{\varrho}\left(\tilde{y}_{s} \notin K_{n} \mid L\right) s^{-\frac{d-3}{d-1}} d s,
$$

where

and

$$
G(x):=\left(\alpha_{d-1}\right)^{\frac{-1}{d-1}} \varrho(x)^{\frac{-1}{d-1}} H_{d-1}(x)^{\frac{1}{2(d-1)}}
$$

$$
\lim _{n \rightarrow \infty} n^{\frac{1}{2}} \zeta(y, n)=\alpha_{d-1} \varrho(x)(2\langle u(x), x\rangle)^{\frac{d-1}{2}} H_{d-1}(x)^{-\frac{1}{2}} .
$$

Now we show that, in the domain of integration, $\zeta(y, n)$ can be replaced by $n^{-1 / 2}$, that is,

$$
J_{\varrho}(y, L)=(d-1)^{-1} G(x)^{2} \lim _{n \rightarrow \infty} \int_{0}^{n^{-1 / 2}} n^{\frac{2}{d-1}} \mathbb{P}_{\varrho}\left(\tilde{y}_{s} \notin K_{n} \mid L\right) s^{-\frac{d-3}{d-1}} d s .
$$

It follows from Lemma 2.1 and (3.4) that there exist constants $c_{0}>0$ and $c_{2}>$ $c_{1}>0$ depending on $y, K, L, \varrho$ such that if $s>0$ is small enough, then

$$
\mathbb{P}_{\varrho}\left(\tilde{y}_{s} \notin K_{n} \mid L\right) \ll\left(1-c_{0} s\right)^{n},
$$

and if $n$ is large and $s$ is between $\zeta(n, y)$ and $n^{-1 / 2}$, then $c_{1} n^{-1 / 2}<s<c_{2} n^{-1 / 2}$. In particular,

$$
\begin{aligned}
& \lim _{n \rightarrow \infty} \int_{c_{1} n^{-1 / 2}}^{c_{2} n^{-1 / 2}} n^{\frac{2}{d-1}} \mathbb{P}_{\varrho}\left(\tilde{y}_{s} \notin K_{n} \mid L\right) s^{-\frac{d-3}{d-1}} d s \\
& \ll \lim _{n \rightarrow \infty} n^{\frac{2}{d-1}} \int_{c_{1} n^{-1 / 2}}^{c_{2} n^{-1 / 2}} e^{-c_{0} n s} s^{-\frac{d-3}{d-1}} d s \\
& \leq \lim _{n \rightarrow \infty} c_{2} n^{\frac{2}{d-1}-\frac{1}{2}} e^{-c_{1} c_{0} n^{\frac{1}{2}}} c_{1}^{-\frac{d-3}{d-1}} n^{\frac{d-3}{2(d-1)}}=0,
\end{aligned}
$$

and hence (3.5) yields (3.6). 
Let $\pi: \mathbb{R}^{d} \rightarrow u(x)^{\perp}$ denote the orthogonal projection to $u(x)^{\perp}$. Using (2.5), (2.3) and (3.4), we obtain

$$
\begin{aligned}
\lim _{s \rightarrow 0^{+}} s^{\frac{-1}{d-1}}\left\|\pi\left(x-\tilde{x}_{s}\right)\right\| & =0, \\
\lim _{s \rightarrow 0^{+}} s^{\frac{-2}{d-1}}\left\langle u(x), x-\tilde{x}_{s}\right\rangle & =\frac{1}{2} G(x)^{2} .
\end{aligned}
$$

Let $Q$ denote the second fundamental form of $\partial K$ at $x$ (cf. (1.1)), considered as a function on $u(x)^{\perp}$. Then there are an orthonormal basis $v_{1}, \ldots, v_{d-1}$ of $u(x)^{\perp}$ and positive numbers $k_{1}, \ldots, k_{d-1}>0$ such that

$$
Q\left(\sum_{i=1}^{d-1} z_{i} v_{i}\right)=\sum_{i=1}^{d-1} k_{i} z_{i}^{2}
$$

Further, let $\pi$ be the orthogonal projection to $u(x)^{\perp}$, and define

$$
E:=\left\{z \in u(x)^{\perp}: Q(z) \leq 1\right\},
$$

which is the Dupin indicatrix of $K$ at $x$, whose half-axes are $k_{i}(x)^{-1 / 2}, i=1, \ldots, d-$ 1. In addition, let $\Gamma$ be the convex hull of the osculating paraboloid of $K$ at $x \in \partial K$, that is,

$$
\Gamma=\left\{x+z-t u(x): z \in u(x)^{\perp}, t \geq \frac{1}{2} Q(z)\right\}
$$

Hence, we have

$$
\Gamma \cap H(x, t)=x_{t}^{*}+\sqrt{2 t\langle x, u(x)\rangle} E,
$$

and there exists an increasing function $\tilde{\mu}(s)$ with $\lim _{s \rightarrow 0^{+}} \tilde{\mu}(s)=1$ such that

$$
\tilde{x}_{s}^{*}+\tilde{\mu}(s)^{-1} G(x) \cdot s^{\frac{1}{d-1}} E \subset K \cap \tilde{H}(x, s) \subset \tilde{x}_{s}^{*}+\tilde{\mu}(s) G(x) \cdot s^{\frac{1}{d-1}} E,
$$

where $\tilde{x}_{s}^{*}:=x_{t}^{*} \in\left(x-\mathbb{R}_{+} u(x)\right) \cap \widetilde{H}(x, s)$, and $s$ and $t$ are related by equation (3.1). From (3.7) it follows that also

$$
\tilde{x}_{s}+\tilde{\mu}(s)^{-1} G(x) \cdot s^{\frac{1}{d-1}} E \subset K \cap \widetilde{H}(x, s) \subset \tilde{x}_{s}+\tilde{\mu}(s) G(x) \cdot s^{\frac{1}{d-1}} E .
$$

The rest of the proof is devoted to identifying the asymptotic behavior of the integral (3.6). First, we adjust the domain of integration and the integrand in a suitable way. In a second step, the resulting expression is compared to the case where $K$ is the unit ball. We recall that $x_{1}, \ldots, x_{n}$ are random points in $\partial K$, and we put $\Xi_{n}:=\left\{x_{1}, \ldots, x_{n}\right\}$, hence $K_{n}=\left[\Xi_{n}\right]$. For a finite set $X \subset \mathbb{R}^{d}$, let $\# X$ denote the cardinality of $X$.

Lemma 3.1. For $\varepsilon \in(0,1)$, there exist $\alpha, \beta>1$ and an integer $k>d$, depending only on $\varepsilon$ and $d$, with the following property. If $L \in \mathcal{L}_{j}^{d}, y \in \partial(K \mid L), x \in \partial K$ is a normal boundary point of $K$ such that $y=x \mid L$ and $H_{d-1}(x)>0$, and if $n>n_{0}$, where $n_{0}$ depends on $\varepsilon, x, K, \varrho, L$, then

$$
\int_{0}^{n^{-1 / 2}} \mathbb{P}_{\varrho}\left(\tilde{y}_{s} \notin K_{n} \mid L\right) s^{-\frac{d-3}{d-1}} d s=\int_{\frac{\varepsilon(d-1) / 2}{n}}^{\frac{\alpha}{n}} \varphi(K, L, y, \varrho, \varepsilon, s) s^{-\frac{d-3}{d-1}} d s+O\left(\frac{\varepsilon}{n^{\frac{2}{d-1}}}\right),
$$

where

$\varphi(K, L, y, \varrho, \varepsilon, s)=\mathbb{P}_{\varrho}\left(\left(\tilde{y}_{s} \notin\left(\left[\widetilde{C}(x, \beta s) \cap \Xi_{n}\right] \mid L\right)\right)\right.$ and $\left.\left(\#\left(\widetilde{C}(x, \beta s) \cap \Xi_{n}\right) \leq k\right)\right)$. 
Proof. Let $\varepsilon \in(0,1)$ be given. Then $\alpha>1$ is chosen such that

$$
2^{d-1+\frac{2 d}{d-1}} \int_{2^{-d} \alpha}^{\infty} e^{-r} r^{\frac{2}{d-1}-1} d r<\varepsilon .
$$

Further, we choose $\beta \geq\left(16^{2}(d-1)\right)^{d-1}$ such that

$$
2^{d-1} e^{-2^{-3 d+2} \sqrt{\beta} \cdot \varepsilon^{\frac{d-1}{2}}}<\varepsilon \cdot \alpha^{\frac{-2}{d-1}},
$$

and then we fix an integer $k>d$ such that

$$
\frac{(\alpha \beta)^{k}}{k !}<\frac{\varepsilon}{\alpha^{\frac{2}{d-1}}} .
$$

Lemma 3.1 follows from the following three statements, which we will prove assuming that $n$ is sufficiently large:

(i) $\int_{0}^{n^{-1 / 2}} \mathbb{P}_{\varrho}\left(\tilde{y}_{s} \notin K_{n} \mid L\right) s^{-\frac{d-3}{d-1}} d s=\int_{\frac{\varepsilon}{n}(d-1) / 2}^{\frac{\alpha}{n}} \mathbb{P}_{\varrho}\left(\tilde{y}_{s} \notin K_{n} \mid L\right) s^{-\frac{d-3}{d-1}} d s+O\left(\frac{\varepsilon}{n^{\frac{2}{d-1}}}\right)$.

(ii) If $\varepsilon^{(d-1) / 2} / n<s<\alpha / n$, then

$$
\mathbb{P}_{\varrho}\left(\#\left(\widetilde{C}(x, \beta s) \cap \Xi_{n}\right) \geq k\right) \leq \frac{\varepsilon}{\alpha^{\frac{2}{d-1}}} .
$$

(iii) If $\varepsilon^{(d-1) / 2} / n<s<\alpha / n$, then

$$
\mathbb{P}_{\varrho}\left(\tilde{y}_{s} \notin K_{n} \mid L\right)=\mathbb{P}_{\varrho}\left(\tilde{y}_{s} \notin\left[\left(\widetilde{C}(x, \beta s) \cap \Xi_{n}\right) \mid L\right]\right)+O\left(\frac{\varepsilon}{\alpha^{\frac{2}{d-1}}}\right) .
$$

Before proving (i), (ii) and (iii), we note that they imply

$$
\begin{aligned}
\int_{0}^{n^{-1 / 2}} \mathbb{P}_{\varrho}\left(\tilde{y}_{s} \notin K_{n} \mid L\right) s^{-\frac{d-3}{d-1}} d s & =\int_{\frac{\varepsilon(d-1) / 2}{n}}^{\frac{\alpha}{n}} \varphi(K, L, y, \varrho, \varepsilon, s) s^{-\frac{d-3}{d-1}} d s \\
& +O\left(\frac{\varepsilon}{\alpha^{\frac{2}{d-1}}}\right) \int_{\frac{\varepsilon(d-1) / 2}{n}}^{\frac{\alpha}{n}} s^{-\frac{d-3}{d-1}} d s+O\left(\frac{\varepsilon}{n^{\frac{2}{d-1}}}\right),
\end{aligned}
$$

which in turn yields Lemma 3.1 .

First, we introduce some notation. As before, let $Q$ be the second fundamental form at $x \in \partial K$, and let $v_{1}, \ldots, v_{d-1}$ be an orthonormal basis of $u(x)^{\perp}$ representing the principal directions. In addition, let $\Theta_{1}^{\prime}, \ldots, \Theta_{2^{d-1}}^{\prime}$ be the corresponding coordinate corners, and for $i=1, \ldots, 2^{d-1}$ and $s \in\left(0, n^{-1 / 2}\right)$, let

$$
\widetilde{\Theta}_{i, s}=\widetilde{C}(x, s) \cap\left(\tilde{x}_{s}+\left[\Theta_{i}^{\prime}, \mathbb{R}_{+} x\right]\right) .
$$

Subsequently, we show that

$$
\lim _{s \rightarrow 0^{+}} s^{-1} \int_{\widetilde{\Theta}_{i, s} \cap \partial K} \varrho(z) \mathcal{H}^{d-1}(d z)=2^{-(d-1)} .
$$

In fact, since a ball rolls freely inside $K, \varrho$ is continuous and positive at $x$, and by (3.7) we deduce that

$$
\begin{aligned}
& \lim _{s \rightarrow 0^{+}} s^{-1} \int_{\widetilde{\Theta}_{i, s} \cap \partial K} \varrho(z) \mathcal{H}^{d-1}(d z) \\
& =\varrho(x) \lim _{s \rightarrow 0^{+}} s^{-1} \mathcal{H}^{d-1}\left(\widetilde{\Theta}_{i, s} \cap \partial K\right) \\
& =\varrho(x) \lim _{s \rightarrow 0^{+}} s^{-1} \mathcal{H}^{d-1}\left(\partial K \cap \widetilde{C}(x, s) \cap\left(\tilde{x}_{s}^{*}+\left[\Theta_{i}^{\prime}, \mathbb{R}_{+} u(x)\right]\right)\right) .
\end{aligned}
$$


Let $\Psi: \partial \Gamma \cap C(x, r / R) \rightarrow \partial K \cap C(x, r / R)$ be the diffeomorphism which assigns to a point $z \in \partial \Gamma \cap \widetilde{H}(x, s)$ the unique point $\Psi(z) \in \partial K \cap\left(\tilde{x}_{s}^{*}+\mathbb{R}_{+}\left(z-\tilde{x}_{s}^{*}\right)\right)$. It follows from (3.7) that there exists an increasing function $\mu: \mathbb{R}_{+} \rightarrow \mathbb{R}_{+}$with $\lim _{s \rightarrow 0^{+}} \mu(s)=1$ such that

$$
\mu(s)^{-1} \leq \operatorname{Lip}(\psi \mid(\partial \Gamma \cap \widetilde{C}(x, s))) \leq \mu(s) .
$$

Thus we get

$$
\begin{aligned}
& \lim _{s \rightarrow 0^{+}} s^{-1} \mathcal{H}^{d-1}\left(\partial K \cap \widetilde{C}(x, s) \cap\left(\tilde{x}_{s}^{*}+\left[\Theta_{i}^{\prime}, \mathbb{R}_{+} u(x)\right]\right)\right) \\
& =\lim _{s \rightarrow 0^{+}} s^{-1} \mathcal{H}^{d-1}\left(\Psi\left(\partial \Gamma \cap \widetilde{C}(x, s) \cap\left(\tilde{x}_{s}^{*}+\left[\Theta_{i}^{\prime}, \mathbb{R}_{+} u(x)\right]\right)\right)\right) \\
& =\lim _{s \rightarrow 0^{+}} s^{-1} \mathcal{H}^{d-1}\left(\partial \Gamma \cap \widetilde{C}(x, s) \cap\left(\tilde{x}_{s}^{*}+\left[\Theta_{i}^{\prime}, \mathbb{R}_{+} u(x)\right]\right)\right) \\
& =2^{-(d-1)} \lim _{s \rightarrow 0^{+}} s^{-1} \mathcal{H}^{d-1}(\partial \Gamma \cap \widetilde{C}(x, s)) .
\end{aligned}
$$

Now we can repeat the preceding argument in reverse order and finally use (3.1) to arrive at the assertion (3.13).

To prove (i), we observe that

$$
\int_{0}^{\frac{\varepsilon^{(d-1) / 2}}{n}} \mathbb{P}_{\varrho}\left(\tilde{y}_{s} \notin K_{n} \mid L\right) s^{-\frac{d-3}{d-1}} d s \leq \int_{0}^{\frac{\varepsilon^{(d-1) / 2}}{n}} s^{-\frac{d-3}{d-1}} d s \ll \frac{\varepsilon}{n^{\frac{2}{d-1}}} .
$$

Let $\alpha / n<s<n^{-1 / 2}$, and let $n$ be sufficiently large. First, (2.6) yields that

$$
\mathbb{P}_{\varrho}\left(o \notin K_{n}, \tilde{y}_{s} \notin K_{n} \mid L\right) \leq \varepsilon n^{-\frac{2}{d-1}} .
$$

On the other hand, if $o \in K_{n}$, then $\tilde{y}_{s} \notin K_{n} \mid L$ implies that $\widetilde{\Theta}_{i, s} \cap K_{n}=\emptyset$ for some $i \in\left\{1, \ldots, 2^{d-1}\right\}$, and hence (3.13) yields

$$
\mathbb{P}_{\varrho}\left(o \in K_{n}, \tilde{y}_{s} \notin K_{n} \mid L\right) \leq 2^{d-1}\left(1-2^{-d} s\right)^{n}<2^{d-1} e^{-2^{-d} n s} .
$$

Therefore, by (3.10) we get

$$
\begin{aligned}
\int_{\alpha / n}^{n^{-1 / 2}} \mathbb{P}_{\varrho}\left(\tilde{y}_{s} \notin K_{n} \mid L\right) s^{-\frac{d-3}{d-1}} d s & \ll 2^{d-1} \int_{\alpha / n}^{\infty} e^{-2^{-d} n s} s^{\frac{2}{d-1}-1} d s+\frac{\varepsilon}{n^{\frac{2}{d-1}}} \\
& =\frac{2^{d-1+\frac{2 d}{d-1}}}{n^{\frac{2}{d-1}}} \int_{2^{-d} \alpha}^{\infty} e^{-r} r^{\frac{2}{d-1}-1} d r+\frac{\varepsilon}{n^{\frac{2}{d-1}}} \\
& \leq \frac{2 \varepsilon}{n^{\frac{2}{d-1}}},
\end{aligned}
$$

which verifies (i).

Next (ii) simply follows from (3.1) and (3.12). In fact, if $0<s<\alpha / n$, then

$$
\mathbb{P}_{\varrho}\left(\#\left(\widetilde{C}(x, \beta s) \cap \Xi_{n}\right) \geq k\right) \leq\left(\begin{array}{l}
n \\
k
\end{array}\right)(\beta s)^{k} \leq\left(\begin{array}{l}
n \\
k
\end{array}\right)\left(\frac{\alpha \beta}{n}\right)^{k}<\frac{(\alpha \beta)^{k}}{k !} \leq \frac{\varepsilon}{\alpha^{\frac{2}{d-1}}} .
$$

Finally, we prove (iii). To this end, if $\varepsilon^{(d-1) / 2} / n<s<\alpha / n$ and $i \in\left\{1, \ldots, 2^{d-1}\right\}$, then we define $w_{i} \in \Theta_{i}^{\prime}$ by

$$
w_{i}:=(\sqrt{\beta} s)^{\frac{1}{d-1}} \sum_{m=1}^{d-1} \frac{\eta_{m} G(x)}{4 \sqrt{(d-1) k_{m}(x)}} v_{m},
$$


where $\eta_{m}=\eta_{m}^{i} \in\{-1,1\}$ for $m=1, \ldots, 2^{d-1}$. Now let

$$
\widetilde{\Omega}_{i, s}:=\partial K \cap\left[\tilde{x}_{s}+\Theta_{i}^{\prime}, \tilde{x}_{\sqrt{\beta} s}+w_{i}+\Theta_{i}^{\prime}\right] .
$$

We claim that for large $n$, if $\tilde{y}_{s} \in K_{n} \mid L$ but $\tilde{y}_{s} \notin\left[\left(\widetilde{C}(x, \beta s) \cap \Xi_{n}\right) \mid L\right]$, then there exists $i \in\left\{1, \ldots, 2^{d-1}\right\}$ such that

$$
\Xi_{n} \cap \widetilde{\Omega}_{i, s}=\emptyset .
$$

Moreover, for all $i=1, \ldots, 2^{d-1}$, we have

$$
\int_{\widetilde{\Omega}_{i, s}} \varrho(z) \mathcal{H}^{d-1}(d z) \geq 2^{-3 d+2} \sqrt{\beta} s .
$$

To justify (3.17), let $i \in\left\{1, \ldots, 2^{d-1}\right\}$ be fixed. It follows from the definition of $w_{i}$ that

$$
w_{i} \in(\sqrt{\beta} s)^{\frac{1}{d-1}} \frac{G(x)}{4} \cdot \partial E .
$$

Recall that $\pi: \mathbb{R}^{d} \rightarrow u(x)^{\perp}$ denotes the orthogonal projection to $u(x)^{\perp}$. If $n$ is large enough, and hence $0<s<\alpha / n$ is sufficiently small, then (3.7), (3.9) and (3.15) yield that $w_{i} \in \pi\left(\widetilde{\Omega}_{i, s}\right)$, since by assumption $\sqrt{\beta}^{1 /(d-1)} / 4>2$, and therefore

$$
\left(w_{i}+\Theta_{i}^{\prime}\right) \cap\left(w_{i}+(\sqrt{\beta} s)^{\frac{1}{d-1}} \frac{G(x)}{4} \cdot E\right) \subset \pi\left(\widetilde{\Omega}_{i, s}\right) .
$$

In particular, (3.17) now follows from

$$
\begin{aligned}
\int_{\widetilde{\Omega}_{i, s}} \varrho(z) \mathcal{H}^{d-1}(d z) & \geq \frac{\varrho(x)}{2} \cdot \mathcal{H}^{d-1}\left(\widetilde{\Omega}_{i, s}\right) \\
& \geq \frac{\varrho(x)}{2} \cdot \mathcal{H}^{d-1}\left(\pi\left(\widetilde{\Omega}_{i, s}\right)\right) \\
& \geq \frac{\varrho(x)}{2} \cdot \frac{1}{2^{d-1}} \sqrt{\beta} s \frac{G(x)^{d-1}}{4^{d-1}} \alpha_{d-1} H_{d-1}(x)^{-1 / 2} \\
& =2^{-d} 4^{1-d} \sqrt{\beta} s .
\end{aligned}
$$

Next we verify (3.16). We assume that $\tilde{y}_{s} \in K_{n} \mid L$ but $\tilde{y}_{s} \notin\left[\left(\widetilde{C}(x, \beta s) \cap \Xi_{n}\right) \mid L\right]$. Then there exist $a \in\left[\left(\widetilde{C}(x, \beta s) \cap \Xi_{n}\right) \mid L\right]$ and $b \in\left(K_{n} \backslash \widetilde{C}(x, \beta s)\right) \mid L$ such that $\tilde{y}_{s} \in(a, b)$. Thus there exists a hyperplane $H$ in $\mathbb{R}^{d}$ containing $\tilde{y}_{s}+L^{\perp}$ and bounding the half-spaces $H^{+}$and $H^{-}$such that $\widetilde{C}(x, \beta s) \cap \Xi_{n} \subset \operatorname{int}\left(H^{+}\right)$and $b \in \operatorname{int}\left(H^{-}\right)$. In addition, there exists $i \in\left\{1, \ldots, 2^{d-1}\right\}$ such that

$$
\tilde{x}_{s}+\Theta_{i}^{\prime} \subset H^{-} \text {. }
$$

Now we define points $q$ and $q^{\prime}$ by

$$
\{q\}=\left[\tilde{y}_{s}, b\right] \cap \widetilde{H}(x, \sqrt{\beta} s), \quad\left\{q^{\prime}\right\}=\left[\tilde{y}_{s}, b\right] \cap \widetilde{H}(x, \beta s) .
$$

Relation (3.7) implies that

$$
\widetilde{H}(x, \beta s) \cap K \subset \tilde{x}_{\beta s}^{*}+2 G(x)(\beta s)^{\frac{1}{d-1}} E
$$

if $s>0$ is sufficiently small. Arguing as in [4], we obtain that

$$
\left\langle u(x), \tilde{y}_{s}-\tilde{y}_{\beta s}\right\rangle<\frac{\beta^{1 /(d-1)}}{\beta^{1 /(d-1)}-1}\left\langle u(x), \tilde{y}_{\sqrt{\beta} s}-\tilde{y}_{\beta s}\right\rangle
$$


and

which yields (cf. [4])

$$
\frac{\left\|q-\tilde{y}_{\sqrt{\beta} s}\right\|}{\left\|q^{\prime}-\tilde{y}_{\beta s}\right\|}=\frac{\left\langle u(x), \tilde{y}_{s}-\tilde{y}_{\sqrt{\beta} s}\right\rangle}{\left\langle u(x), \tilde{y}_{s}-\tilde{y}_{\beta s}\right\rangle}
$$

$$
q \in \tilde{y}_{\sqrt{\beta} s}+2 s^{\frac{1}{d-1}} G(x) E .
$$

Since $\beta \geq\left[8^{2}(d-1)\right]^{d-1}$, we thus arrive at

$$
q \in \tilde{y}_{\sqrt{\beta} s}+\frac{1}{4 \sqrt{d-1}}(\sqrt{\beta} s)^{\frac{1}{d-1}} G(x) E .
$$

Now (3.18) implies that $q+\Theta_{i}^{\prime} \subset H^{-}$. Hence it follows from (3.19) that $\tilde{y}_{\sqrt{\beta} s}+w_{i} \subset$ $q+\Theta_{i}^{\prime} \subset H^{-}$, and therefore also $\tilde{y}_{\sqrt{\beta} s}+w_{i}+\Theta_{i}^{\prime} \subset H^{-}$. Thus $\widetilde{\Omega}_{i, s} \subset H^{-}$, which yields $\Xi_{n} \cap \widetilde{\Omega}_{i, s}=\emptyset$.

Assertion (iii) follows from (3.16) and (3.17). In fact, if $\varepsilon^{(d-1) / 2} / n<s<\alpha / n$, then

$$
\begin{aligned}
& \mathbb{P}_{\varrho}\left(\tilde{y}_{s} \notin\left[\left(\widetilde{C}(y, \beta s) \cap \Xi_{n}\right) \mid L\right]\right)-\mathbb{P}_{\varrho}\left(\tilde{y}_{s} \notin\left(K_{n} \mid L\right)\right) \\
& \leq \sum_{i=1}^{2^{d-1}}\left(1-\int_{\widetilde{\Omega}_{i, s}} \varrho(z) \mathcal{H}^{d-1}(d z)\right)^{n} \\
& \leq 2^{d-1} e^{-2^{-3 d+2} \sqrt{\beta} \cdot s n} \\
& \leq \varepsilon \alpha^{-\frac{2}{d+1}}
\end{aligned}
$$

by the choice of $\beta$.

To actually compare the situation near the normal boundary point $x$ of $K$ with $H_{d-1}(x)>0$ to the case of the unit ball, let $\sigma=\left(d \alpha_{d}\right)^{-1}$ be the constant density of the corresponding probability distribution on $S^{d-1}$. Let $w \in S^{d-1}$ be the $d$ th coordinate vector in $\mathbb{R}^{d}$, and hence $\mathbb{R}^{d-1}=w^{\perp}$. We write $B_{n}$ to denote the convex hull of $n$ random points distributed uniformly and independently on $S^{d-1}$ according to $\sigma$. For $s \in\left(0, \frac{1}{2}\right)$, we fix a linear subspace $L_{0} \in \mathcal{L}_{j}^{d}$ with $w \in L_{0}$, and let $\tilde{w}_{s}$ be of the form $\lambda w$ for $\lambda \in(0,1)$ such that

$$
\left(d \alpha_{d}\right)^{-1} \cdot \mathcal{H}^{d-1}\left(\left\{z \in S^{d-1}:\langle z, w\rangle \geq\left\langle\tilde{w}_{s}, w\right\rangle\right\}\right)=s .
$$

In particular, $\tilde{w}_{s} \mid L_{0}=\tilde{w}_{s}$.

Lemma 3.2. If $L \in \mathcal{L}_{j}^{d}, y \in \partial(K \mid L)$ and $x \in \partial K$ is a normal boundary point such that $y=x \mid L$ and $H_{d-1}(x)>0$, then

$$
\begin{aligned}
\lim _{n \rightarrow \infty} & \int_{0}^{n^{-1 / 2}} n^{\frac{2}{d-1}} \mathbb{P}_{\varrho}\left(\tilde{y}_{s} \notin K_{n} \mid L\right) s^{-\frac{d-3}{d-1}} d s \\
& =\lim _{n \rightarrow \infty} \int_{0}^{n^{-1 / 2}} n^{\frac{2}{d-1}} \mathbb{P}_{\sigma}\left(\tilde{w}_{s} \notin B_{n} \mid L_{0}\right) s^{-\frac{d-3}{d-1}} d s .
\end{aligned}
$$

Proof. First, we assume $d \geq 3$. It is sufficient to prove that for any $\varepsilon \in(0,1)$ there exists $n_{0}>0$, depending on $\varepsilon, x, K, \varrho, L$, such that if $n>n_{0}$, then

$$
\int_{0}^{n^{-1 / 2}} \mathbb{P}_{\varrho}\left(\tilde{y}_{s} \notin K_{n} \mid L\right) s^{-\frac{d-3}{d-1}} d s=\int_{0}^{n^{-1 / 2}} \mathbb{P}_{\sigma}\left(\tilde{w}_{s} \notin B_{n} \mid L_{0}\right) s^{-\frac{d-3}{d-1}} d s+O\left(\frac{\varepsilon}{n^{\frac{2}{d-1}}}\right) .
$$


Let $\alpha, \beta$ and $k$ be the quantities associated with $\varepsilon, x, K, \varrho, L$ in Lemma 3.1 let $\widetilde{C}(x, s)$ denote the cap of $K$ defined in (3.2), and let $\widetilde{C}(w, s)$ denote the corresponding cap of $B^{d}$ at $w$. We define the densities $\varrho_{s}$ on $\partial \widetilde{C}(x, \beta s)$ and $\sigma_{s}$ on $\partial \widetilde{C}(w, \beta s)$ of probability distributions by

$$
\begin{aligned}
& \varrho_{s}(z)=\left\{\begin{aligned}
\varrho(z) /(\beta s), & \text { if } z \in \partial K \cap \widetilde{C}(x, \beta s), \\
0, & \text { if } z \in \partial \widetilde{C}(x, \beta s) \backslash \partial K,
\end{aligned}\right. \\
& \sigma_{s}(z)=\left\{\begin{aligned}
\sigma(z) /(\beta s), & \text { if } z \in S^{d-1} \cap \widetilde{C}(w, \beta s), \\
0, & \text { if } z \in \partial \widetilde{C}(w, \beta s) \backslash S^{d-1} .
\end{aligned}\right.
\end{aligned}
$$

For $i=0, \ldots, k$, we write $\widetilde{C}(x, \beta s)_{i}$ and $\widetilde{C}(w, \beta s)_{i}$ to denote the convex hulls of $i$ random points distributed uniformly and independently on $\partial \widetilde{C}(x, \beta s)$ and $\partial \widetilde{C}(w, \beta s)$ according to $\varrho_{s}$ and $\sigma_{s}$, respectively.

If $n$ is large, then Lemma 3.1 yields that the left-hand and the right-hand sides of $(3.20)$ are

$$
\begin{gathered}
O\left(\frac{\varepsilon}{n^{\frac{2}{d-1}}}\right)+\sum_{i=0}^{k}\left(\begin{array}{c}
n \\
i
\end{array}\right) \int_{\frac{\varepsilon}{(d-1) / 2}}^{\frac{\alpha}{n}}(\beta s)^{i}(1-\beta s)^{n-i} \mathbb{P}_{\varrho_{s}}\left(\tilde{y}_{s} \notin \widetilde{C}(x, \beta s)_{i} \mid L\right) s^{-\frac{d-3}{d-1}} d s, \\
O\left(\frac{\varepsilon}{n^{\frac{2}{d-1}}}\right)+\sum_{i=0}^{k}\left(\begin{array}{c}
n \\
i
\end{array}\right) \int_{\frac{\varepsilon}{(d-1) / 2}}^{\frac{\alpha}{n}}(\beta s)^{i}(1-\beta s)^{n-i} \mathbb{P}_{\sigma_{s}}\left(\tilde{w}_{s} \notin \widetilde{C}(w, \beta s)_{i} \mid L_{0}\right) s^{-\frac{d-3}{d-1}} d s .
\end{gathered}
$$

For each $i \leq k$, the representation of the beta function by the gamma function and the Stirling formula (see E. Artin [1) implies

$$
\lim _{n \rightarrow \infty} n^{\frac{2}{d-1}}\left(\begin{array}{c}
n \\
i
\end{array}\right) \int_{0}^{1 / \beta}(\beta s)^{i}(1-\beta s)^{n-i} s^{-\frac{d-3}{d-1}} d s=\frac{\beta^{\frac{-2}{d-1}} \Gamma\left(i+\frac{2}{d-1}\right)}{i !}<1 .
$$

Therefore, to prove (3.20), it is sufficient to verify that for each $i=0, \ldots, k$, if $s>0$ is small, then

$$
\left|\mathbb{P}_{\varrho_{s}}\left(\tilde{y}_{s} \notin \widetilde{C}(x, \beta s)_{i} \mid L\right)-\mathbb{P}_{\sigma_{s}}\left(\tilde{w}_{s} \notin \widetilde{C}(w, \beta s)_{i} \mid L_{0}\right)\right| \ll \frac{\varepsilon}{k} .
$$

If $i \leq j$, then (3.22) readily holds as its left-hand side is zero.

To prove (3.22) if $i \in\{j+1, \ldots, k\}$, we transform both $K$ and $B^{d}$ in such a way that their osculating paraboloid is $\Omega=\left\{z-\|z\|^{2} w: z \in \mathbb{R}^{d-1}\right\}$, and the images of the caps $\widetilde{C}(x, \beta s)$ and $\widetilde{C}(w, \beta s)$ are very close. Using these caps, we construct equivalent representations of $\mathbb{P}_{\varrho_{s}}\left(\tilde{y}_{s} \notin \widetilde{C}(x, \beta s)_{i} \mid L\right)$ and $\mathbb{P}_{\sigma_{s}}\left(\tilde{w}_{s} \notin \widetilde{C}(w, \beta s)_{i} \mid L_{0}\right)$, based on the same space $\Xi_{s}$ and on comparable probability measures and random variables.

We may assume that $u(x)=w$. Let $v_{1}, \ldots, v_{d-1}$ be an orthonormal basis of $w^{\perp}$ in the principal directions of the fundamental form $Q$ of $K$ at $x \in \partial K$. We define the linear transform $A_{s}$ of $\mathbb{R}^{d}$ by

$$
\begin{aligned}
& A_{s}(w)=2(\beta s)^{\frac{-2}{d-1}} G(x)^{-2} w, \\
& A_{s}\left(v_{i}\right)=(\beta s)^{\frac{-1}{d-1}} \sqrt{k_{i}(x)} G(x)^{-1} v_{i}, \quad i=1, \ldots, d-1,
\end{aligned}
$$

and choose an orthonormal linear transform $P_{s}$ such that $P_{s} w=w$, and $P_{s} \circ$ $A_{s}\left(L^{\perp}\right)=L_{0}^{\perp}$. Based on these linear transforms, let $\Phi_{s}$ be the affine transformation

$$
\Phi_{s}(z)=P_{s} \circ A_{s}(z-x) .
$$


In addition, we define the linear transform $R_{s}$ of $\mathbb{R}^{d}$ by

$$
\begin{aligned}
& R_{s}(w)=2(\beta s)^{\frac{-2}{d-1}}\left(\frac{\alpha_{d-1}}{d \alpha_{d}}\right)^{\frac{2}{d-1}} w \\
& R_{s}\left(v_{i}\right)=(\beta s)^{\frac{-1}{d-1}}\left(\frac{\alpha_{d-1}}{d \alpha_{d}}\right)^{\frac{1}{d-1}} v_{i}, \quad i=1, \ldots, d-1,
\end{aligned}
$$

and let $\Psi_{s}$ be the affine transformation

$$
\Psi_{s}(z)=R_{s}(z-x) .
$$

Subsequently, we also write $\Phi_{s} z$ for $\Phi_{s}(z)$ or $\Phi_{s} z \mid L_{0}$ for $\Phi_{s}(z) \mid L_{0}$, and similarly for $\Psi_{s}$. We observe that $\Omega$ is the osculating paraboloid of both $\Phi_{s} K$ and $\Psi_{s} B^{d}$ at $o$, and

$$
\lim _{s \rightarrow 0^{+}} \Phi_{s} \tilde{x}_{s}=\lim _{s \rightarrow 0^{+}} \Psi_{s} \tilde{w}_{s}=-\beta^{\frac{-2}{d-1}} w=: w^{*},
$$

$\lim _{s \rightarrow 0^{+}} \Phi_{s} \widetilde{C}(x, \beta s)=\lim _{s \rightarrow 0^{+}} \Psi_{s} \widetilde{C}(w, \beta s)=\left\{z-\tau w: z \in B^{d-1}\right.$ and $\left.\|z\|^{2} \leq \tau \leq 1\right\}$.

For $p \in \widetilde{C}(x, \beta s) \cap \partial K$ and $z=\pi \circ \Phi_{s}(p)$, let $D(p)$ be the Jacobian of $\pi \circ \Phi_{s}$ at $p$ as a map $\pi \circ \Phi_{s}: \widetilde{C}(x, \beta s) \cap \partial K \rightarrow \mathbb{R}^{d-1}$, and let

$$
\tilde{\varrho}_{s}(z)=\varrho_{s}(p) \cdot D(p)^{-1} \text {. }
$$

In addition, for $p \in \widetilde{C}(w, \beta s) \cap S^{d-1}$ and $z=\pi \circ \Psi_{s}(p)$, let $\widetilde{D}(p)$ be the Jacobian of $\pi \circ \Psi_{s}$ at $p$ as a map $\pi \circ \Psi_{s}: \widetilde{C}(w, \beta s) \cap S^{d-1} \rightarrow \mathbb{R}^{d-1}$, and let

$$
\tilde{\sigma}_{s}(z)=\sigma_{s}(p) \cdot \widetilde{D}(p)^{-1} \text {. }
$$

We define

and extend $\tilde{\varrho}_{s}$ and $\tilde{\sigma}_{s}$ to $\Xi_{s}$ by

$$
\Xi_{s}=\left[\pi \circ \Phi_{s} \widetilde{C}(x, \beta s)\right] \cup\left[\pi \circ \Psi_{s} \widetilde{C}(w, \beta s)\right],
$$

$$
\begin{aligned}
& \tilde{\varrho}_{s}(z)=0, \text { if } z \in\left[\pi \circ \Psi_{s} \widetilde{C}(w, \beta s)\right] \backslash\left[\pi \circ \Phi_{s} \widetilde{C}(x, \beta s)\right], \\
& \tilde{\sigma}_{s}(z)=0, \text { if }\left[\pi \circ \Phi_{s} \widetilde{C}(x, \beta s)\right] \backslash\left[\pi \circ \Psi_{s} \widetilde{C}(w, \beta s)\right] .
\end{aligned}
$$

Therefore $\tilde{\varrho}_{s}$ and $\tilde{\sigma}_{s}$ are densities of probability distributions on $\Xi_{s}$. For $z \in \Xi_{s}$, let $\varphi_{s}(z) \in \Phi_{s} \partial K$ and $\psi_{s}(z) \in \Psi_{s} S^{d-1}$ be the points near $z$ whose orthogonal projection into $\mathbb{R}^{d-1}$ is $z$. For random variables $z_{1}, \ldots, z_{i} \in \Xi_{s}$ either with respect to $\tilde{\varrho}_{s}$ or $\tilde{\sigma}_{s}$, the quantities above were defined so as to satisfy

$$
\begin{aligned}
\mathbb{P}_{\varrho_{s}}\left(\tilde{y}_{s} \notin \widetilde{C}(x, \beta s)_{i} \mid L\right) & =\mathbb{P}_{\tilde{\varrho}_{s}}\left(\Phi_{s} \tilde{x}_{s}\left|L_{0} \notin\left[\varphi_{s}\left(z_{1}\right), \ldots, \varphi_{s}\left(z_{i}\right)\right]\right| L_{0}\right), \\
\mathbb{P}_{\sigma_{s}}\left(\tilde{w}_{s} \notin \widetilde{C}(w, \beta s)_{i} \mid L\right) & =\mathbb{P}_{\tilde{\sigma}_{s}}\left(\Psi_{s} \tilde{w}_{s} \notin\left[\psi_{s}\left(z_{1}\right), \ldots, \psi_{s}\left(z_{i}\right)\right] \mid L_{0}\right) .
\end{aligned}
$$

Now there exists an increasing function $s \mapsto \mu^{*}(s)$ with $\lim _{s \rightarrow 0^{+}} \mu^{*}(s)=1$ such that

$$
\mu^{*}(s)^{-1} B^{d-1} \subset\left[\pi \circ \Phi_{s} \widetilde{C}(x, \beta s)\right] \cap\left[\pi \circ \Psi_{s} \widetilde{C}(w, \beta s)\right] \subset \Xi_{s} \subset \mu^{*}(s) B^{d-1},
$$

we have $\mu^{*}(s)^{-1} \varphi_{s}(z) \leq \psi_{s}(z) \leq \mu^{*}(s) \varphi_{s}(z)$ for all $z \in \Xi_{s}$, and

$$
\begin{array}{ll}
\mu^{*}(s)^{-1} \alpha_{d-1}^{-1} \leq \tilde{\varrho}_{s}(z) \leq \mu^{*}(s) \alpha_{d-1}^{-1}, & \text { if } z \in \pi \circ \Phi_{s} \widetilde{C}(x, \beta s), \\
\mu^{*}(s)^{-1} \alpha_{d-1}^{-1} \leq \tilde{\sigma}_{s}(z) \leq \mu^{*}(s) \alpha_{d-1}^{-1}, & \text { if } z \in \pi \circ \Psi_{s} \widetilde{C}(w, \beta s) .
\end{array}
$$


Therefore

$$
\lim _{s \rightarrow 0^{+}} \int_{\Xi_{s}}\left|\varrho_{s}(z)-\tilde{\sigma}_{s}(z)\right| \mathcal{H}^{d-1}(d z)=0 .
$$

From (3.25) we deduce that if $s>0$ is small, then

$$
\begin{gathered}
\mid \mathbb{P}_{\tilde{\varrho}_{s}}\left(\Phi_{s} \tilde{x}_{s}\left|L_{0} \notin\left[\varphi_{s}\left(z_{1}\right), \ldots, \varphi_{s}\left(z_{i}\right)\right]\right| L_{0}\right. \text { and } \\
\left.\Psi_{s} \tilde{w}_{s} \notin\left[\psi_{s}\left(z_{1}\right), \ldots, \psi_{s}\left(z_{i}\right)\right] \mid L_{0}\right) \\
-\mathbb{P}_{\tilde{\sigma}_{s}}\left(\Phi_{s} \tilde{x}_{s}\left|L_{0} \notin\left[\varphi_{s}\left(z_{1}\right), \ldots, \varphi_{s}\left(z_{i}\right)\right]\right| L_{0}\right. \text { and } \\
\left.\Psi_{s} \tilde{w}_{s} \notin\left[\psi_{s}\left(z_{1}\right), \ldots, \psi_{s}\left(z_{i}\right)\right] \mid L_{0}\right) \mid \leq \frac{\varepsilon}{k} .
\end{gathered}
$$

Next, if $s>0$ is small, then

$$
\left\|w^{*}-\Phi_{s} \tilde{x}_{s}\right\| \leq \frac{\varepsilon}{k^{j+1}} \quad \text { and } \quad\left\|w^{*}-\Psi_{s} \tilde{w}_{s}\right\| \leq \frac{\varepsilon}{k^{j+1}},
$$

and in addition,

$$
\left\|\varphi_{s}(z)-\psi_{s}(z)\right\| \leq \frac{\varepsilon}{k^{j+1}} \quad \text { for all } z \in \Xi_{s} .
$$

Let us assume that $\Phi_{s} \tilde{x}_{s}\left|L_{0} \notin\left[\varphi_{s}\left(z_{1}\right), \ldots, \varphi_{s}\left(z_{i}\right)\right]\right| L_{0}$ but $\Psi_{s} \tilde{w}_{s} \in\left[\psi_{s}\left(z_{1}\right), \ldots\right.$, $\left.\psi_{s}\left(z_{i}\right)\right] \mid L_{0}$ for some $z_{1}, \ldots, z_{i} \in \Xi_{s}$. In this case, the point $a$ of $\left[\varphi_{s}\left(z_{1}\right), \ldots, \varphi_{s}\left(z_{i}\right)\right] \mid L_{0}$ closest to $\Phi_{s} \tilde{x}_{s} \mid L_{0}$ is contained in some $(j-1)$-simplex $\left[\varphi_{s}\left(z_{m_{1}}\right), \ldots, \varphi_{s}\left(z_{m_{j}}\right)\right] \mid L_{0}$, i.e., there are $\lambda_{1}, \ldots, \lambda_{j} \geq 0, \lambda_{1}+\cdots+\lambda_{j}=1$, such that $a=\sum_{r=1}^{j} \lambda_{r} \varphi\left(z_{m_{r}}\right) \mid L_{0}$. There are $\mu_{1}, \ldots, \mu_{i} \geq 0, \mu_{1}+\cdots+\mu_{i}=1$, so that $\Psi_{s} \tilde{w}_{s}=\sum_{r=1}^{i} \mu_{r} \psi_{s}\left(z_{r}\right) \mid L_{0}$. Then we have

$$
\begin{aligned}
\left\|\Phi_{s} \tilde{x}_{s} \mid L_{0}-a\right\| & \leq\left\|\Phi_{s} \tilde{x}_{s}\left|L_{0}-\sum_{r=1}^{i} \mu_{r} \varphi_{s}\left(z_{r}\right)\right| L_{0}\right\| \\
& \leq\left\|\Phi_{s} \tilde{x}_{s}\left|L_{0}-w^{*}\|+\| w^{*}-\Psi_{s} \tilde{w}_{s}\|+\| \sum_{r=1}^{i} \mu_{r}\left(\psi_{s}\left(z_{r}\right)-\varphi_{s}\left(z_{r}\right)\right)\right| L_{0}\right\| \\
& \leq \frac{\varepsilon}{k^{j+1}}+\frac{\varepsilon}{k^{j+1}}+\frac{\varepsilon}{k^{j+1}}=\frac{3 \varepsilon}{k^{j+1}},
\end{aligned}
$$

and hence

$$
\left\|w^{*}-a\right\| \leq \frac{4 \epsilon}{k^{j+1}} .
$$

Choose a maximal set $v_{1}, \ldots, v_{l} \in S^{d-1} \cap L_{0}$ such that the distance between any two points is at least $\varepsilon k^{-(j+1)}$, in particular,

$$
l \ll \varepsilon^{-(j-1)} k^{(j-1)(j+1)} .
$$

Since $a, \varphi_{s}\left(z_{m_{1}}\right)\left|L_{0}, \ldots, \varphi_{s}\left(z_{m_{j}}\right)\right| L_{0}$ lie in a $(j-1)$-dimensional affine subspace of $L_{0}$, there is a unit vector $v \in S^{d-1} \cap L_{0}$ such that $\left|\left\langle\varphi_{s}\left(z_{m_{r}}\right)-w^{*}, v\right\rangle\right| \leq 4 \varepsilon k^{-(j+1)}$ for $r=1, \ldots, j$, and thus

$$
\left|\left\langle\varphi_{s}\left(z_{m_{r}}\right)-w^{*}, v_{m}\right\rangle\right| \leq \frac{6 \varepsilon}{k^{j+1}}
$$

for $r=1, \ldots, j$ and a suitably chosen $m \in\{1, \ldots, l\}$. In fact, for the given vector $v \in S^{d-1} \cap L_{0}$, there is some $m \in\{1, \ldots, l\}$ such that $\left\|v-v_{m}\right\| \leq \varepsilon k^{-(j+1)}$. Since 
$\Phi_{s} \widetilde{C}(x, \beta s) \subset w^{*}+2 B^{d}$, we deduce that

$$
\begin{aligned}
\left|\left\langle\varphi_{s}\left(z_{m_{r}}\right)-w^{*}, v_{m}\right\rangle\right| & \leq\left|\left\langle\varphi_{s}\left(z_{m_{r}}\right)-w^{*}, v\right\rangle\right|+\left\|\varphi_{s}\left(z_{m_{r}}\right)-w^{*}\right\| \cdot\left\|v_{m}-v\right\| \\
& \leq \frac{4 \epsilon}{k^{j+1}}+2 \cdot \frac{\epsilon}{k^{j+1}}=\frac{6 \epsilon}{k^{j+1}} .
\end{aligned}
$$

Therefore, if we define, for $m=1, \ldots, l$,

$$
\Pi_{m}:=\left\{p \in \partial \Phi_{s} \widetilde{C}(x, \beta s):\left|\left\langle p-w^{*}, v_{m}\right\rangle\right| \leq 6 \varepsilon k^{-(j+1)}\right\},
$$

we get the following: If $\Phi_{s} \tilde{x}_{s}\left|L_{0} \notin\left[\varphi_{s}\left(z_{1}\right), \ldots, \varphi_{s}\left(z_{i}\right)\right]\right| L_{0}$ but $\Psi_{s} \tilde{w}_{s} \in\left[\psi_{s}\left(z_{1}\right), \ldots\right.$, $\left.\psi_{s}\left(z_{i}\right)\right] \mid L_{0}$ for some $z_{1}, \ldots, z_{i} \in \Xi_{s}$, then there exists $m \in\{1, \ldots, l\}$ such that $\Pi_{m}$ contains some $j$ of the points $\varphi_{s}\left(z_{1}\right), \ldots, \varphi_{s}\left(z_{i}\right)$. Since $\mathcal{H}^{d-1}\left(\Pi_{m}\right) \ll \varepsilon k^{-(j+1)}$, we have

$$
\begin{aligned}
& \mathbb{P}_{\tilde{\varrho}_{s}}\left(\Phi_{s} \tilde{x}_{s}\left|L_{0} \notin\left[\varphi_{s}\left(z_{1}\right), \ldots, \varphi_{s}\left(z_{i}\right)\right]\right| L_{0} \text { and } \Psi_{s} \tilde{w}_{s} \in\left[\psi_{s}\left(z_{1}\right), \ldots, \psi_{s}\left(z_{i}\right)\right] \mid L_{0}\right) \\
& \quad \leq\left(\begin{array}{c}
i \\
j
\end{array}\right) \sum_{m=1}^{l} \mathbb{P}_{\tilde{\varrho}_{s}}\left(\varphi_{s}\left(z_{1}\right), \ldots, \varphi_{s}\left(z_{j}\right) \in \Pi_{m}\right) \\
& \quad \ll\left(\begin{array}{c}
i \\
j
\end{array}\right) \cdot l \cdot\left(\varepsilon k^{-(j+1)}\right)^{j} \ll \frac{\varepsilon}{k} .
\end{aligned}
$$

Similarly, we have

$$
\mathbb{P}_{\tilde{\sigma}_{s}}\left(\Psi_{s} \tilde{w}_{s} \notin\left[\psi_{s}\left(z_{1}\right), \ldots, \psi_{s}\left(z_{i}\right)\right] \mid L_{0} \text { and } \Phi_{s} \tilde{x}_{s}\left|L_{0} \in\left[\varphi_{s}\left(z_{1}\right), \ldots, \varphi_{s}\left(z_{i}\right)\right]\right| L_{0}\right) \ll \frac{\varepsilon}{k} \text {. }
$$

Combining (3.23), (3.24) as well as (3.26), (3.27) and (3.28) yields (3.22), and in turn Lemma 3.2 if $d \geq 3$.

If $d=2$, then a similar argument works; only some of the constrains should be modified as follows. In (3.21), we only have $\beta^{\frac{-2}{d-1}} \Gamma\left(i+\frac{2}{d-1}\right) / i !<k+1$, and hence in (3.22), we should verify an upper bound of order $\frac{\varepsilon}{k^{2}}$, not of order $\frac{\varepsilon}{k}$. Therefore the upper bound in (3.26) should be $\frac{\varepsilon}{k^{2}}$.

\section{Completing the Proof of Theorem 1.2}

In order to transfer an integral over an average of projections of a convex body to a boundary integral, we are going to use the following lemma from K. Böröczky Jr., L. M. Hoffmann, and D. Hug [3].

For $L \in \mathcal{L}_{j}^{d}$ and $y \in \partial(K \mid L)$, we choose a point $x(y) \in \partial K$ such that $y=x(y) \mid L$. In general, $x(y)$ is not uniquely determined, but we can fix a measurable choice (cf. [3. p. 152]). Recall, however, that $x(y)$ is uniquely determined for $\nu_{j}$ a.e. $L \in \mathcal{L}_{j}^{d}$ and $\mathcal{H}^{j-1}$ a.e. $y \in \partial(K \mid L)$.

Lemma 4.1. Let $K \subset \mathbb{R}^{d}$ be a convex body in which a ball rolls freely, let $f: \partial K \rightarrow$ $[0, \infty)$ be nonnegative and measurable, and let $j \in\{1, \ldots, d-1\}$. Then

$$
\frac{j \alpha_{j}}{d \alpha_{d}} \int_{\partial K} f(x) H_{d-j}(x) \mathcal{H}^{d-1}(d x)=\int_{\mathcal{L}_{j}^{d}} \int_{\partial(K \mid L)} f(x(y)) \mathcal{H}^{j-1}(d y) \nu_{j}(d L) .
$$

By the very special case $K=B^{d}$ of (1.2), due to M. Reitzner [16], we have

$$
\lim _{n \rightarrow \infty} n^{\frac{2}{d-1}}\left[V_{j}\left(B^{d}\right)-\mathbb{E}_{\sigma} V_{j}\left(B_{n}\right)\right]=c^{(j, d)}\left(d \alpha_{d}\right)^{\frac{d+1}{d-1}} .
$$


Therefore the rotational symmetry of $B^{d}, 2.12$ and (3.6) yield

$$
\begin{aligned}
c^{(j, d)}\left(d \alpha_{d}\right)^{\frac{d+1}{d-1}}= & \frac{\left(\begin{array}{c}
d \\
j
\end{array}\right) \alpha_{d}}{\alpha_{d-j} \alpha_{j}} \cdot \frac{j \alpha_{j}\left(d \alpha_{d}\right)^{\frac{2}{d-1}}}{d-1}\left(\alpha_{d-1}\right)^{-\frac{2}{d-1}} \\
& \times \lim _{n \rightarrow \infty} \int_{0}^{n^{-1 / 2}} n^{\frac{2}{d-1}} \mathbb{P}_{\sigma}\left(\tilde{w}_{s} \notin B_{n} \mid L_{0}\right) s^{-\frac{d-3}{d-1}} d s .
\end{aligned}
$$

We can now transform the asymptotic formulas to $K$. Let $L \in \mathcal{L}_{j}^{d}$ and let $y \in \partial(K \mid L)$ be such that $y=x \mid L$ for some normal boundary point $x=x(y) \in \partial K$. If $H_{d-1}(x)=0$, then $J_{\varrho}(y, L)=0$ by Lemma 2.4. If $H_{d-1}(x)>0$, then it follows from (3.6), Lemma 3.2 and (4.1) that

$$
\begin{aligned}
J_{\varrho}(y, L)= & (d-1)^{-1}\left(\alpha_{d-1}\right)^{-\frac{2}{d-1}} \varrho(x)^{\frac{-2}{d-1}} H_{d-1}(x)^{\frac{1}{d-1}} \\
& \times \lim _{n \rightarrow \infty} \int_{0}^{n^{-1 / 2}} n^{\frac{2}{d-1}} \mathbb{P}_{\sigma}\left(\tilde{w}_{s} \notin B_{n} \mid L_{0}\right) s^{-\frac{d-3}{d-1}} d s \\
= & c^{(j, d)} \varrho(x)^{\frac{-2}{d-1}} H_{d-1}(x)^{\frac{1}{d-1}}\left(\frac{\left(\begin{array}{c}
d \\
j
\end{array}\right) \alpha_{d}}{\alpha_{d-j} \alpha_{j}} \cdot \frac{j \alpha_{j}}{d \alpha_{d}}\right)^{-1},
\end{aligned}
$$

where $x=x(y)$. Finally, we first apply (2.12), and afterwards Lemma 4.1, to deduce

$$
\begin{aligned}
& \lim _{n \rightarrow \infty} n^{\frac{2}{d-1}}\left[V_{j}(K)-\mathbb{E}_{\varrho}\left(V_{j}\left(K_{n}\right)\right)\right] \\
& =c^{(j, d)} \frac{d \alpha_{d}}{j \alpha_{j}} \int_{\mathcal{L}_{j}^{d}} \int_{\partial(K \mid L)} \varrho(x(y))^{\frac{-2}{d-1}} H_{d-1}(x(y))^{\frac{1}{d-1}} \mathcal{H}^{j-1}(d y) \nu_{j}(d L) \\
& =c^{(j, d)} \int_{\partial K} \varrho(x)^{\frac{-2}{d-1}} H_{d-1}(x)^{\frac{1}{d-1}} H_{d-j}(x) \mathcal{H}^{d-1}(d x),
\end{aligned}
$$

which concludes the proof of Theorem 1.2

\section{Proof of Theorem 1.3}

Using the Stirling formula $\Gamma(n+1) \sim\left(\frac{n}{e}\right)^{n} \sqrt{2 \pi n}$, as $n \rightarrow \infty$ (see E. Artin [1]), for any $\alpha>0$ and $\gamma \in(0,1]$, we deduce

$$
\begin{aligned}
\lim _{n \rightarrow \infty} n^{\alpha} \int_{0}^{\gamma} s^{\alpha-1}(1-s)^{n} d s & =\lim _{n \rightarrow \infty} n^{\alpha} \int_{0}^{1} s^{\alpha-1}(1-s)^{n} d s \\
& =\lim _{n \rightarrow \infty} n^{\alpha} \frac{\Gamma(\alpha) \Gamma(n+1)}{\Gamma(n+1+\alpha)}=\Gamma(\alpha) .
\end{aligned}
$$

In the following argument, $\gamma_{1}, \gamma_{2}, \ldots$ again denote positive constants that may depend on $K$ and $\varrho$. We can assume that $o \in \operatorname{int}(K)$. Further, let $(\partial K)_{*}^{n}$ denote the set of all $x_{1}, \ldots, x_{n} \in \partial K$ such that $o \in\left[x_{1}, \ldots, x_{n}\right]$. For $u \in S^{d-1}$ and $t \geq 0$, let

$$
C(u, t):=\left\{x \in K:\langle x, u\rangle \geq h_{K}(u)-t\right\},
$$


where $h_{K}$ denotes the support function of $K$. To deduce the upper bound, we start with the estimates

$$
\begin{aligned}
& \mathbb{E}_{\varrho}\left(V_{1}(K)-V_{1}\left(K_{n}\right)\right) \\
& =\frac{1}{\alpha_{d-1}} \int_{(\partial K)^{n}} \int_{S^{d-1}}\left(h_{K}(u)-h_{K_{n}}(u)\right) \mathcal{H}^{d-1}(d u) \varrho\left(x_{1}\right) \ldots \\
& \varrho\left(x_{n}\right) \mathcal{H}^{d-1}\left(d x_{1}\right) \ldots \mathcal{H}^{d-1}\left(d x_{n}\right) \\
& \leq \frac{1}{\alpha_{d-1}} \int_{(\partial K)_{*}^{n}} \int_{S^{d-1}}\left(h_{K}(u)-h_{K_{n}}(u)\right) \mathcal{H}^{d-1}(d u) \varrho\left(x_{1}\right) \ldots \\
& \varrho \frac{1}{\alpha_{d-1}} \int_{S^{d-1}} \int_{0}^{h_{K}(u)} \int_{\left.(\partial K)^{n}\right) \mathcal{H}^{d-1}\left(d x_{1}\right) \ldots \mathcal{H}^{d-1}\left(d x_{n}\right)+2^{d}\left(1-\gamma_{1}\right)^{n}} \mathbf{1}\left\{x_{1}, \ldots, x_{n} \in \partial K \backslash C(u, s)\right\} \varrho\left(x_{1}\right) \cdots \varrho\left(x_{n}\right) \\
& \leq \frac{1}{\alpha_{d-1}} \int_{S^{d-1}} \int_{0}^{h_{K}(u)}\left(1-\int_{\partial K \cap C(u, t)} \mathcal{H}^{d-1}\left(d x_{1}\right) \ldots \mathcal{H}^{d-1}\left(d x_{n}\right) d s \mathcal{H}^{d-1}(d u)+2^{d}\left(1-\gamma_{1}\right)^{n}\right. \\
& d-1(d x))^{n} d t \mathcal{H}^{d-1}(d u)
\end{aligned}
$$

For suitable positive constants $\gamma_{2}, \gamma_{3}, \gamma_{4}$ we get, for $u \in S^{d-1}$ and $t \in\left(0, \gamma_{2}\right)$,

$$
\int_{\partial K \cap C(u, t)} \varrho(x) \mathcal{H}^{d-1}(d x)>\left\{\begin{array}{cl}
\gamma_{3} t^{d-1}, & \text { if } t \in\left(0, \gamma_{2}\right), \\
\gamma_{4}, & \text { if } t \geq \gamma_{2} .
\end{array}\right.
$$

In particular, $\gamma_{4}, \gamma_{3}\left(\gamma_{2}\right)^{d-1} \in(0,1)$. We deduce from (5.2), (5.3) and (5.1) that, for suitable $\gamma_{5}, \ldots, \gamma_{9}$ with $\gamma_{7}, \gamma_{9} \in(0,1)$,

$$
\begin{aligned}
\mathbb{E}_{\varrho}\left(V_{1}(K)-V_{1}\left(K_{n}\right)\right) & \leq \gamma_{5} \int_{0}^{\gamma_{2}}\left(1-\gamma_{3} t^{d-1}\right)^{n} d t+\gamma_{6} \gamma_{7}^{n} \\
& =\gamma_{8} \int_{0}^{\gamma_{9}} s^{\frac{1}{d-1}-1} \cdot(1-s)^{n} d s+\gamma_{6} \gamma_{7}^{n} \leq \gamma_{10} n^{\frac{-1}{d-1}}
\end{aligned}
$$

To prove the lower bound for $\mathbb{E}_{\varrho}\left(V_{1}(K)-V_{1}\left(K_{n}\right)\right)$, we need the following observation.

Lemma 5.1. Let $K \subset \mathbb{R}^{d}$ be a convex body, and let $h_{K}$ be twice differentiable at $u_{0} \in S^{d-1}$. Then there is some $R>0$ such that $K \subset x_{0}-R u_{0}+R B^{d}$, where $x_{0}=\nabla h_{K}\left(u_{0}\right) \in \partial K$. In particular, there exist a measurable set $\Sigma \subset S^{d-1}$ with $\mathcal{H}^{d-1}(\Sigma)>0$ and some $R>0$, all depending on $K$, such that for any $u \in \Sigma$ there is some $x \in \partial K$ such that $K \subset x-R u+R B^{d}$.

Proof. For the proof of the first assertion, we may assume that $x_{0}=o$; we have hence $h_{K}\left(u_{0}\right)=0$. We put $h:=h_{K}$. By assumption, there is a function $R: \mathbb{R}_{+} \rightarrow$ $[0, \infty)$ with $\lim _{t \rightarrow 0^{+}} R(t)=0$ and

$$
\left|h(u)-\frac{1}{2} \cdot d^{2} h\left(u-u_{0}, u-u_{0}\right)\right| \leq R\left(\left\|u-u_{0}\right\|\right)\left\|u-u_{0}\right\|^{2} .
$$

Thus there is a constant $R_{1}>0$ and $\delta>0$ such that $h(u) \leq R_{1}\left\|u-u_{0}\right\|^{2}$ for all $u \in S^{d-1}$ with $\left\langle u, u_{0}\right\rangle \geq 1-\delta$. For $R_{2}:=\max \left\{2 R_{1}, \max \left\{h(u): u \in S^{d-1}\right\} /(2 \delta)\right\}$ 
and all $u \in S^{d-1}$, we obtain

$$
h(u) \leq R_{2}\left(1-\left\langle u_{0}, u\right\rangle\right)=h\left(-R_{2} u_{0}+R_{2} B^{d}, u\right),
$$

that is, $K \subset-R_{2} u_{0}+R_{2} B^{d}$.

The second assertion follows immediately from the first assertion.

Let $t_{0}$ be the inradius of $K$. Now Lemma 5.1 yields, for $u \in \Sigma$ and $t \in\left(0, t_{0}\right)$, that

$$
\int_{\partial K \cap C(u, t)} \varrho(x) \mathcal{H}^{d-1}(d x)<\gamma_{11} \cdot t^{\frac{d-1}{2}} .
$$

Choosing a constant $\gamma_{12} \in\left(0, t_{0}\right)$ satisfying $\gamma_{11}\left(\gamma_{12}\right)^{\frac{d-1}{2}}<1$, it follows as in the derivation of (5.2) that, with a suitable constant $\gamma_{13} \in(0,1)$, we have

$$
\begin{aligned}
\mathbb{E}_{\varrho}\left(V_{1}(K)-V_{1}\left(K_{n}\right)\right) & \geq \frac{1}{\alpha_{d-1}} \int_{\Sigma} \int_{0}^{\gamma_{12}}\left(1-\gamma_{11} t^{\frac{d-1}{2}}\right)^{n} d t \mathcal{H}^{d-1}(d x) \\
& =\int_{0}^{\gamma_{13}} s^{\frac{2}{d-1}-1} \cdot(1-s)^{n} d s>\gamma_{14} \cdot n^{\frac{-2}{d-1}} .
\end{aligned}
$$

Theorem 1.2 shows that the lower bound of Lemma 1.3 is of optimal order if $K$ has a rolling ball. In fact, the assumption of a rolling ball ensures that the integral on the right side of (1.2) is positive. This follows, for instance, from the absolute continuity of the Gauss curvature measure of a convex body which has a rolling ball (cf. [12]).

On the other hand, the upper bound for $\mathbb{E}_{\varrho}\left(V_{1}(K)-V_{1}\left(K_{n}\right)\right)$ is of optimal order if $K$ is a polytope. To explain this, let $\Sigma_{0} \subset S^{n-1}$ be contained in the interior of the exterior normal cone of one of the vertices of $K$ and such that $\mathcal{H}^{d-1}\left(\Sigma_{0}\right)>0$. In this case

$$
\int_{\partial K \cap C(u, t)} \varrho(x) \mathcal{H}^{d-1}(d x)<\gamma_{15} \cdot t^{d-1}
$$

for $u \in \Sigma_{0}$ and $t \in\left(0, \gamma_{16}\right)$, and hence $\mathbb{E}_{\varrho}\left(V_{1}(K)-V_{1}\left(K_{n}\right)\right) \geq \gamma_{17} \cdot n^{\frac{-1}{d-1}}$.

\section{References}

1. E. Artin: The gamma function. Holt, Rinehart and Winston, 1964. MR0165148 (29:2437)

2. I. Bárány, D.G. Larman: Convex bodies, economic cap coverings, random polytopes. Mathematika 35 (1988), 274-291. MR986636 (90c:52020)

3. K. Böröczky Jr., L.M. Hoffmann, D. Hug: Expectation of intrinsic volumes of random polytopes. Period. Math. Hungar. 57 no. 2 (2008), 143-164. MR.2469601 (2009k:52006)

4. K. Böröczky Jr., F. Fodor, D. Hug: The mean width of random polytopes circumscribed around a convex body. J. London Math. Soc. 81 (2010), 499-523. MR2603007 (2011b:52007)

5. K. Böröczky Jr., M. Reitzner: Approximation of smooth convex bodies by random circumscribed polytopes. Ann. Applied Probab. 14 (2004), 239-273. MR2023022 (2004i:60014)

6. K. Böröczky Jr., R. Schneider: The mean width of circumscribed random polytopes. Canadian Math. Bull. 53 (2010), 614-628. MR2761364

7. H. Federer: Geometric Measure Theory. Springer, Berlin, 1969. MR0257325 (41:1976)

8. P.M. Gruber: Convex and Discrete Geometry. Fundamental Principles of Math. Sci., 336, Springer, Berlin 2007. MR2335496 (2008f:52001)

9. D. Hug: Contributions to affine surface area. Manuscripta Math. 91 (1996), no. 3, 283-301. MR.1416712 (98d:52009)

10. D. Hug: Curvature relations and affine surface area of convex sets. Results Math. 29 (1996), 233-248. MR1387565 (97c:52004)

11. D. Hug: Absolute continuity for curvature measures of convex sets I. Math. Nachr. 195 (1998), 139-158. MR.1654685 (99j:52002) 
12. D. Hug: Absolute continuity for curvature measures of convex sets, II. Math. Z. 232 (1999), 437-485. MR1719698 (2000m:52009)

13. D. Hug: Absolute continuity for curvature measures of convex sets, III. Advances Math. 169 (2002), 92-117. MR1916372(2003e:52004)

14. D. Hug: Measures, Curvatures and Currents in Convex Geometry. Habilitationsschrift, Universität Freiburg, 2000.

15. K. Leichtweiss: Affine geometry of convex bodies. Johann Ambrosius Barth Verlag, 1998. MR.1630116 (2000j:52005)

16. M. Reitzner: Random points on the boundary of smooth convex bodies. Trans. Amer. Math. Soc. 354, no. 6 (2002), 2243-2278. MR 1885651 (2002m:52003)

17. R. Schneider: Approximation of convex bodies by random polytopes. Aequationes Math. 32 (1987), 304-310. MR900710 (88j:52012)

18. R. Schneider: Convex Bodies: the Brunn-Minkowski Theory. Cambridge University Press, Cambridge, 1993. MR1216521 (94d:52007)

19. C. Schütt: Random polytopes and affine surface area. Math. Nach. 170 (1994), 227-249. MR1302377 (95k:52007)

20. C. Schütt, E. Werner: The convex floating body. Math. Scand. 66 (1990), 275-290. MR.1075144 (91i:52005)

21. C. Schütt, E. Werner: Polytopes with vertices chosen randomly from the boundary of a convex body. Geometric aspects of functional analysis, 241-422, Lecture Notes in Math., 1807, Springer, Berlin, 2003. MR2083401 (2005g:52015)

Alfréd Rényi Institute of Mathematics, Hungarian Academy of Sciences, Reáltanoda

U. 13-15, 1053 Budapest, Hungary

E-mail address: carlos@renyi.hu

Department of Geometry, University of Szeged, Aradi vértanúk tere 1, H-6720 Szeged, Hungary - And - Department of Mathematics and Statistics, University of CalGary, 2500 University Dr. N.W., Calgary, Alberta, Canada T2N 1N4

E-mail address: fodorf@math.u-szeged.hu

Karlsruhe Institute of Technology, Department of Mathematics, D-76128 KarlSRuhe, Germany

E-mail address: daniel.hug@kit.edu 